\title{
Heavy Higgs of the Twin Higgs models
}

\author{
Aqeel Ahmed \\ Faculty of Physics, University of Warsaw, \\ Pasteura 5, Warsaw, 02-093 Poland \\ PRISMA Cluster of Excellence $\&$ Mainz Institute for Theoretical Physics, \\ Johannes Gutenberg University, Mainz, 55099 Germany \\ Laboratoire de Physique Théorique, CNRS, Université Paris-Sud 11, \\ Orsay Cedex, F-91405 France \\ E-mail: aqeel.ahmed@fuw.edu.pl
}

ABSTRACT: Twin Higgs models are the prime illustration of neutral naturalness, where the new particles of the twin sector, gauge singlets of the Standard Model (SM), ameliorate the little hierarchy problem. In this work, we analyse phenomenological implications of the heavy Higgs of the Mirror Twin Higgs and Fraternal Twin Higgs models, when electroweak symmetry breaking is linearly realized. The most general structure of twin Higgs symmetry breaking, including explicit soft and hard breaking terms in the scalar potential, is employed. The direct and indirect searches at the LHC are used to probe the parameter space of Twin Higgs models through mixing of the heavy Higgs with the SM Higgs and decays of the heavy Higgs to the SM states. Moreover, for the Fraternal Twin Higgs, we study the production and decays of twin glueball and bottomonium states to the SM light fermions, which have interesting signatures involving displaced vertices and are potentially observable at the colliders.

Keywords: Beyond Standard Model, Higgs Physics

ArXIV EPRINT: 1711.03107 


\section{Contents}

1 Introduction 1

2 Electroweak symmetry breaking in Twin Higgs models 4

2.1 Physical basis for Twin Higgs models $\quad 7$

2.2 Electroweak precision constraints in Twin Higgs models 8

3 Heavy Higgs phenomenology in Mirror Twin Higgs 9

4 Heavy Higgs phenomenology in Fraternal Twin Higgs $\quad 15$

$\begin{array}{lll}4.1 & \text { Fraternal Twin hadron production and decays } & 18\end{array}$

$\begin{array}{lll}4.2 & \text { Fraternal Twin hadron phenomenology } & 22\end{array}$

4.3 Heavy Twin Higgs phenomenology 25

5 Conclusions $\quad 25$

$\begin{array}{ll}\text { A Twin Higgs Feynman rules and partial decay widths } & 27\end{array}$

\section{Introduction}

The discovery of a scalar boson with mass $125 \mathrm{GeV}$ at the Large Hadron Collider (LHC) and its properties show that it is consistent with the Higgs boson of the Standard Model (SM). The SM of particle physics is the most accurate theory of elementary particles and their interactions, however, it is not a complete theory of nature since there are many unanswered puzzles which go beyond the Standard Model (BSM). In particular, the discovery of a relatively light SM Higgs has highlighted the need to address naturalness/hierarchy problem more than ever before, i.e. there should be some mechanism which cancels the SM radiative corrections to the Higgs mass. Motivated by naturalness, enormous work has been done in the last few decades constructing BSM scenarios. Most of the BSM models including supersymmetry, composite Higgs, and extra dimensions predict some sort of new physics (top partners) just above the electroweak scale to cancel the quantum corrections to the Higgs mass. However, the absence of such new physics signatures at the LHC pose a "little hierarchy" (fine-tuning) problem. Hence, there is a need to construct and analyse new BSM scenarios which can explain the elusiveness of the top-partners and solve the SM puzzles.

Neutral Naturalness is one such class of models, where the top-partners are not charged under the SM gauge symmetry, so they are very hard to produce at the colliders. Twin Higgs (TH) model [1-4] is the prime and first example of neutral naturalness, which is designed to resolve the little hierarchy problem and make the top-partners elusive. In a nutshell, TH models have two core ingredients: (i) the SM-like Higgs is a Goldstone boson 
which emerges due to spontaneous breaking of a global symmetry, hence its mass is protected, and (ii) the twin top partners are neutral ("colorless") under the SM gauge group, which explains their elusiveness at the LHC. In twin Higgs mechanism the cancellations of radiative corrections to the SM Higgs mass are due to the exchange of new twin states which are related to the SM by a discrete $\mathbb{Z}_{2}$ symmetry.

The simplest realization of twin Higgs mechanism [1] employs an SU(4) global symmetry which is spontaneously broken to $\mathrm{SU}(3)$ at a high energy scale $\Lambda$ and gives $\mathbf{7}$ Goldstone bosons and a radial mode. A subgroup $\mathrm{SU}(2) \times \widehat{\mathrm{SU}}(2)$ of $\mathrm{SU}(4)$ global symmetry is gauged, where $\mathrm{SU}(2)$ and $\widehat{\mathrm{SU}}(2)$ represent the $\mathrm{SM}$ and twin weak gauge groups, respectively (hat "^" denotes twin sector). After TH electroweak symmetry breaking (EWSB), 6 of the Goldstone bosons of SU(4)/SU(3) coset are "eaten" by the weak gauge bosons of the SM $\left(W^{ \pm}, Z\right)$ and twin $\left(\hat{W}^{ \pm}, \hat{Z}\right)$ sectors. The remaining one Goldstone boson serves as the SM Higgs. The original incarnation of this idea, where the twin sector has an exact mirror copy of the SM particle content, is referred as 'Mirror Twin Higgs' (MTH) model [1].

However, in order to manifest the features of the twin Higgs mechanism, one does not need to have an exact copy of the SM particle content in the twin sector, but rather a minimal number of states are necessary to ensure the cancellation of radiative corrections to the SM Higgs mass. This possibility was first discussed in the so-called 'Fraternal Twin Higgs' (FTH) model [5]. In order to solve the little hierarchy problem, the FTH model requires only the third generation of quarks and leptons, and the weak gauge bosons in the twin sector. Another striking difference of the FTH model to the MTH case is twin hadron spectroscopy in the two models. In particular, the FTH model has two interesting features: (i) unlike the MTH model, there are no fraternal twin hadrons with light quark flavors $(\hat{u}, \hat{d}, \hat{s}, \hat{c})$, but the fraternal twin hadrons are glueball $[\hat{g} \hat{g}]$ and bottomonium $[\hat{b} \hat{b}]$ states [5], and (ii) the fraternal twin QCD confinement scale, $\hat{\Lambda}_{\mathrm{QCD}}$, can be much larger than that of the MTH model, which is due to fewer twin quark flavors and faster running of the twin QCD couplings $\hat{\alpha}_{\mathrm{QCD}}$. Hence, the fraternal twin hadrons (glueballs/bottomoniums) can be relatively heavier [5]. Note that the twin hadrons are colorless under the SM QCD. The lightest stable twin hadrons in the MTH model are the mirror baryons which could be dark matter candidates [2], however, in the FTH case the stable twin hadronic states are the lightest glueball/bottomonium states [5]. Moreover, among the fraternal twin hadrons, the $0^{++}$glueball and bottomonium states have the correct quantum numbers to mix with the scalar sector of the model. ${ }^{1}$ This mixing leads to exotic decays of the fraternal $0^{++}$twin hadrons back into the SM light quarks and leptons at the colliders [5]. These features of the FTH model are similar to those of the 'hidden valley' models [6-11].

There are many variants of TH models which offer UV complete description of the TH mechanism; including supersymmetric TH [12-18], composite/holographic TH [1924], and some more recent $\mathrm{TH}$ constructions [25-35]. In most of the TH models, it is assumed that the radial mode of TH EWSB is of the order of symmetry breaking scale or heavier. Hence the radial mode is integrated out and low-energy effective theory contains

\footnotetext{
${ }^{1}$ The $0^{++}$twin hadrons are also present in the MTH model, however, given the usual spectroscopy of mirror twin hadrons, these states have low production multiplicity and are unstable (similar to SM $0^{++}$ hadrons). Hence, phenomenologically they have less impact than the fraternal $0^{++}$twin hadrons.
} 
only the Goldstone bosons. However, if the TH spontaneous symmetry breaking is linearly realized then, in principle, the radial mode can be much lighter than the symmetry breaking scale and can be present in the low-energy theory, ${ }^{2}$ in fact, is favored by the electroweak precisions tests (EWPT) (see below, section 2.2). Note that the TH symmetry breaking can be linearly realized in a perturbative UV complete TH model, e.g. supersymmetric TH [16]. However, we work in an effective field theory (EFT) framework of the TH models without considering any specific perturbative UV complete scenario. In this work, we explore the scalar sector of the TH mechanism with a focus on the phenomenological implications of the radial mode. The twin radial mode is heavier than the SM-like Higgs of mass $125 \mathrm{GeV}$, hence we refer it as 'heavy' twin Higgs.

Twin Higgs scalar sector provides a portal between the visible (SM) and invisible (twin) sectors. Hence, the discovery potential of twin Higgs mechanism needs a detailed phenomenological analysis of the scalar sector of TH models. Recently, there has been some work done on different aspects of the TH scalar sector involving only the SM Higgs, see e.g. [5, 25-33, 39-42]. In this work, we aim to investigate the phenomenological aspects of the scalar sector of MTH and FTH models focusing on heavy twin Higgs. We parametrize the fundamental scalar of SU(4), which breaks the global symmetry, by two Higgs doublets $H$ and $\widehat{H}$. We consider the most general form of TH scalar potential with soft and hard $\mathbb{Z}_{2}$ discrete and $\mathrm{SU}(4)$ global symmetry breaking terms. The effective TH scalar potential has five parameters, however, after fixing the SM-like Higgs mass $m_{h}=125 \mathrm{GeV}$ and its vacuum expectation value (vev) $v=246 \mathrm{GeV}$, we are left with three free parameters; the heavy twin Higgs mass, its vev, and the hard $\mathbb{Z}_{2}$ breaking parameter. In this work, we explore the parameter space spanned by these three free parameters of the TH models at the LHC. Note that relatively small number of free parameters offers a unique opportunity to discover the twin Higgs mechanism at the current and/or future colliders.

To analyse phenomenological aspects of the scalar sector of the MTH and FTH models, we calculate the branching fractions of the heavy Higgs to the SM and dark (twin) states. It turns out that dominant decay channels of heavy twin Higgs are pairs of the SM Higgs, SM weak gauge bosons, and twin weak gauge bosons (if kinematically allowed), roughly with equal branching fractions. This makes the heavy Higgs decays to the SM massive gauge bosons and the SM Higgs as the prime channels to discover the TH models at the colliders. We perform a detailed analysis of these channels at the LHC with $14 \mathrm{TeV}$ centerof-mass energy. Moreover, indirect searches at the LHC, via the SM Higgs signal-strength measurements and its invisible decay width, provide a complementary probe to the scalar sector of the TH models.

In the case of FTH, the phenomenological study of the twin hadrons (twin glueball and bottomonium states) is much more involved, mainly due to the lack of proper knowledge of twin QCD confinement/hadronization. However, for our purposes, we make conservative estimates of the twin hadron production through the heavy twin Higgs. The production rates for the twin $0^{++}$hadrons through heavy twin Higgs are about the same order as

\footnotetext{
${ }^{2} \mathrm{~A}$ possibility of twin Higgs radial mode is also discussed in [2, 5, 16, 36]. Moreover, see [37, 38] for the phenomenology of the radial mode in composite Higgs model.
} 
those of the SM Higgs and massive gauge bosons. These twin $0^{++}$hadrons (glueball and/or bottomonium states) decay back to the SM light quarks and leptons through the Higgs mixing, see a prototype process in figure 1 . The $0^{++}$twin hadrons decays are either prompt or displaced depending on their kinematics. Especially, the displaced signals offer a unique discovery potential for the FTH model at the colliders.

The paper is organized as follows. Twin Higgs symmetry breaking details, the choice of physical basis, and EWPT constraints on TH models are presented in section 2. The phenomenological analysis of the twin Higgs of the MTH and FTH models are presented in sections 3 and 4, respectively. Section 5 contains the summary and conclusions. We collect supplementary material in appendix A, which includes Feynman rules and partial decay widths of the heavy twin Higgs to the SM and twin sector states.

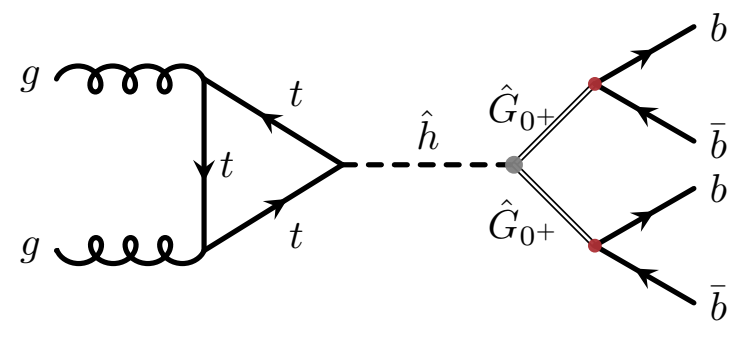

Figure 1. An illustration of a benchmark process of $0^{++}$twin glueball $\hat{G}_{0^{+}}$production via heavy twin Higgs $\hat{h}$ at the LHC and decays to the SM bottom quarks through an off-shell SM Higgs.

\section{Electroweak symmetry breaking in Twin Higgs models}

We consider the TH models where an SU(4) global symmetry is spontaneously broken to $\mathrm{SU}(3)$ providing 7 Goldstone bosons. For the TH symmetry breaking we employ a scalar $\mathbb{H}$ which belongs to fundamental representation of $\mathrm{SU}(4)$ and parametrize as,

$$
\mathbb{H}=\frac{1}{\sqrt{2}} \exp \left(\frac{i \Pi}{f_{0}}\right)\left(\begin{array}{c}
0 \\
0 \\
0 \\
\mathcal{H}
\end{array}\right),
$$

where $\Pi$ is a matrix containing the $\mathbf{7}$ Goldstone bosons and $\mathcal{H}$ is a radial Higgs which acquires a vev $\langle\mathcal{H}\rangle \equiv f_{0}$. Twin Higgs potential for the EWSB is

$$
V(\mathbb{H})=\lambda\left(\mathbb{H}^{\dagger} \mathbb{H}-f_{0}^{2} / 2\right)^{2},
$$

where using (2.1), we get a quartic potential for the radial Higgs $\mathcal{H}$,

$$
V(\mathcal{H})=\frac{1}{4} \lambda\left(\mathcal{H}^{2}-f_{0}^{2}\right)^{2} .
$$

In order to have a global minimum of the above potential we require,

$$
\left.\frac{d V(\mathcal{H})}{d \mathcal{H}}\right|_{\mathcal{H}=f_{0}}=0, \quad \text { and }\left.\quad \frac{d^{2} V(\mathcal{H})}{d \mathcal{H}^{2}}\right|_{\mathcal{H}=f_{0}}>0
$$

The above conditions insure that mass squared of the radial fluctuation, $m_{\hat{h}_{0}}^{2}=2 \lambda f_{0}^{2}$, is positive for $f_{0}^{2}>0$ and $\lambda>0$. Note that mass of the radial mode can be much lower than 
the TH symmetry breaking scale $f_{0}$ for $\lambda<1$. Whereas for $\lambda \gg 1$, we recover a non-linear sigma model version of the twin Higgs models where the radial mode is decoupled.

In this work, we parametrize the scalar $\mathbb{H}$ in a linear sigma model framework, i.e.

$$
\mathbb{H}=\left(\begin{array}{c}
H \\
\widehat{H}
\end{array}\right), \quad H \equiv \frac{1}{\sqrt{2}}\left(\begin{array}{c}
h_{1}+i h_{2} \\
v+h_{0}+i h_{3}
\end{array}\right), \quad \widehat{H} \equiv \frac{1}{\sqrt{2}}\left(\begin{array}{c}
\hat{h}_{1}+i \hat{h}_{2} \\
\hat{v}+\hat{h}_{0}+i \hat{h}_{3}
\end{array}\right)
$$

where $H$ and $\widehat{H}$ are doublets under the SM and twin SU(2) weak groups, respectively, whereas $v$ and $\hat{v}$ are their respective vevs. The most general TH effective scalar potential including explicit soft and hard $\mathbb{Z}_{2}$ discrete and SU(4) global symmetry breaking terms can be written as $[5,16]$,

$$
V_{\text {eff }}(H, \widehat{H})=\lambda\left(|H|^{2}+|\widehat{H}|^{2}-\frac{f_{0}^{2}}{2}\right)^{2}+\kappa\left(|H|^{4}+|\widehat{H}|^{4}\right)-\sigma f_{0}^{2}|H|^{2}+\rho|H|^{4} .
$$

Note that one can rewrite all other possible $\mathbb{Z}_{2} / \mathrm{SU}(4)$ explicit symmetry breaking terms in the form of above potential parameters. As mentioned above, we do not aim to consider any specific UV complete description of TH models, rather we assume that the above form of effective potential can arise from a particular UV complete model, e.g. [16].

Before moving forward, we would like to discuss the role/significance of each term in the above effective potential.

1. The $\lambda$-term is invariant under $\mathrm{SU}(4)$ global symmetry as well as respects $\mathbb{Z}_{2}$ discrete symmetry under which $H \leftrightarrow \widehat{H}$. As noted above, for $f_{0}^{2}>0$, this terms is the source of spontaneous breaking of SU(4) global symmetry to $\mathrm{SU}(3)$ giving $\mathbf{7}$ Goldstone bosons plus a radial mode $\hat{h}_{0}$.

2. The $\kappa$-term is invariant under the discrete $\mathbb{Z}_{2}$ symmetry, however, breaks $\mathrm{SU}(4)$ global symmetry explicitly. For $\kappa>0$, the vevs $v$ and $\hat{v}$ are degenerate in the absence of $\mathbb{Z}_{2}$ breaking term, i.e. $v=\hat{v}=f_{0} / \sqrt{(2 \lambda+\kappa)}$, which is phenomenologically not a viable option.

3. The $\sigma$-term 'softly' breaks both $\mathrm{SU}(4)$ global and $\mathbb{Z}_{2}$ discrete symmetries, as a result a misalignment of the two vevs is generated.

4. The $\rho$-term captures 'hard' breaking effects of $\mathbb{Z}_{2} / \mathrm{SU}(4)$ symmetries and it helps to reduce the fine-tuning in TH models [16].

Note that the $\mathbb{Z}_{2} / \mathrm{SU}(4)$ breaking terms are important in the above potential to generate mass of the SM-like Higgs boson, which would have been a pure Goldstone boson in the absence of such terms. Moreover, these explicit symmetry breaking parameters are expected to be smaller than the symmetry preserving parameter, i.e. $(\kappa, \sigma, \rho) \leq \lambda$, in order to not produce large radiative corrections to the SM-like Higgs mass.

We impose unitary gauge where 6 Goldstone bosons are "eaten" by the weak gauge bosons of the $\mathrm{SM}\left(W^{ \pm}, Z\right)$ and twin $\left(\hat{W}^{ \pm}, \hat{Z}\right)$ sectors. Hence, in the unitary gauge, we 
write the SM Higgs $H$ and twin Higgs $\widehat{H}$ doublets as,

$$
H=\frac{1}{\sqrt{2}}\left(\begin{array}{c}
0 \\
v+h_{0}
\end{array}\right), \quad \widehat{H}=\frac{1}{\sqrt{2}}\left(\begin{array}{c}
0 \\
\hat{v}+\hat{h}_{0}
\end{array}\right) .
$$

Minimization of the scalar potential (2.5) results in the following values of vevs, $v^{2}$ and $f^{2} \equiv v^{2}+\hat{v}^{2}$

$$
v^{2}=\frac{f_{0}^{2}(\kappa \lambda+\sigma(\kappa+\lambda))}{\rho(\kappa+\lambda)+\kappa(\kappa+2 \lambda)}, \quad f^{2}=\frac{f_{0}^{2}(\kappa(2 \lambda+\sigma)+\lambda \rho)}{\rho(\kappa+\lambda)+\kappa(\kappa+2 \lambda)} .
$$

We define a parameter $\xi \equiv v / f$. Roughly speaking, $2 \xi^{2}$ measures the amount of fine-tuning in our model. ${ }^{3}$ In a limit $\kappa \ll \lambda$, the $\xi^{2}$ can be written as,

$$
\xi^{2} \simeq \frac{\sigma}{\rho}+\frac{\kappa(\rho(\lambda+\sigma)-\sigma(2 \lambda+\sigma))}{\lambda \rho^{2}}+\mathcal{O}\left(\frac{\kappa^{2}}{\lambda^{2}}\right) .
$$

In order to have small fine-tuning in the model, i.e. $\xi^{2} \ll 1$ or $v^{2} \ll f^{2}$, one needs a tuning in the parameters $\sigma$ and $\rho$, such that $\sigma \ll \rho$ for fixed $\lambda$ and $\kappa$. This result implies that large hard breaking effects could potentially lower the amount of fine-tuning in TH models, see also a similar discussion in [16].

Moving on, the mass-squared matrix for the scalar fluctuations $\left(h_{0}, \hat{h}_{0}\right)$ is,

$$
\mathcal{M}^{2}=\left(\begin{array}{cc}
2 v^{2}(\lambda+\kappa+\rho) & 2 \lambda v \hat{v} \\
2 \lambda v \hat{v} & 2 \hat{v}^{2}(\lambda+\kappa)
\end{array}\right) .
$$

We diagonalize $\mathcal{M}^{2}$ by an orthogonal rotational matrix $\mathcal{R}$ as

$$
\mathcal{M}_{\text {diag }}^{2} \equiv \mathcal{R}^{-1} \mathcal{M}^{2} \mathcal{R}=\left(\begin{array}{cc}
m_{h}^{2} & 0 \\
0 & m_{\hat{h}}^{2}
\end{array}\right), \quad \text { with } \quad \mathcal{R}=\left(\begin{array}{cc}
\cos \alpha & \sin \alpha \\
-\sin \alpha & \cos \alpha
\end{array}\right),
$$

where $(h, \hat{h})$ are the two scalar mass-eigenstates (without the subscript ' 0 ') with masses $\left(m_{h}^{2}, m_{\hat{h}}^{2}\right)$. The two physical scalars, SM Higgs $h$ and heavy twin Higgs $\hat{h}$, are admixture of $h_{0}$ and $\hat{h}_{0}$, and are defined as

$$
h=h_{0} \cos \alpha-\hat{h}_{0} \sin \alpha, \quad \hat{h}=h_{0} \sin \alpha+\hat{h}_{0} \cos \alpha .
$$

The corresponding mass eigenvalues are,

$$
m_{h}^{2}=\rho v^{2}+(\lambda+\kappa) f^{2}(1-\Delta), \quad m_{\hat{h}}^{2}=\rho v^{2}+(\lambda+\kappa) f^{2}(1+\Delta),
$$

where

$$
\Delta^{2}=1-\frac{1}{\lambda+\kappa} \frac{v^{2}}{f^{2}}\left[\frac{4\left(\kappa^{2}+2 \lambda \kappa\right)}{\lambda+\kappa}\left(1+\frac{v^{2}}{f^{2}}\right)+2 \rho\left(1+2 \frac{v^{2}}{f^{2}}\right)+\rho^{2} \frac{v^{2}}{f^{2}}\right] .
$$

\footnotetext{
${ }^{3}$ We do not discuss in detail, issues related to the fine-tuning in $\mathrm{TH}$ models but refer the interested readers to a dedicated recent paper [43], and also [5, 16].
} 
The mixing angle is given by

$$
\tan (2 \alpha)=\frac{2 \xi \sqrt{1-\xi^{2}}}{\left(1-(2+\rho / \lambda) \xi^{2}\right)+\left(1-2 \xi^{2}\right) \kappa / \lambda}
$$

For more intuition of the above results, we expand in the limit $\xi^{2}=v^{2} / f^{2} \ll 1$. At the leading order in this approximation, we get the mass eigenvalues as,

$$
m_{h}^{2}=2 \rho v^{2}+\frac{2 v^{2}\left(\kappa^{2}+2 \kappa \lambda\right)}{\kappa+\lambda}, \quad m_{\hat{h}}^{2}=2 f^{2}(\kappa+\lambda)-\frac{2 v^{2}\left(\kappa^{2}+2 \kappa \lambda\right)}{\kappa+\lambda} .
$$

The above expressions manifestly show that in the limit $\xi^{2} \ll 1$ and fixed $v^{2}$, the SM Higgs mass is directly related to hard breaking parameter $\rho$. Therefore, the hard breaking parameter $\rho$ would be severely constrained by the SM Higgs mass. However, it is possible to have sizable $\rho$, in order to reduce the fine-tuning as mentioned above in eq. (2.9), but at the price of reducing $\kappa$ to get the SM Higgs mass correct, see also [16]. Furthermore, in the same approximation $\xi^{2} \ll 1$, the mixing angle can be written as,

$$
\sin ^{2} \alpha=\frac{\lambda^{2}}{(\lambda+\kappa)^{2}} \frac{v^{2}}{f^{2}}+\mathcal{O}\left(\frac{v^{4}}{f^{4}}\right)
$$

which shows a dependence of $\sin ^{2} \alpha \simeq v^{2} / f^{2}$ similar to composite Higgs model.

Before going ahead, we would like to comment on the most relevant scalar sector couplings, the triple Higgs couplings $g_{h h h}$ and $g_{\hat{h} h h}$, which are given as

$$
\begin{aligned}
& g_{h h h}=6\left[v \cos \alpha\left(\lambda+(\kappa+\rho) \cos ^{2} \alpha\right)-\hat{v} \sin \alpha\left(\lambda+\kappa \sin ^{2} \alpha\right)\right], \\
& g_{\hat{h} h h}=2\left[v \sin \alpha\left(\lambda+3(\kappa+\rho) \cos ^{2} \alpha\right)+\hat{v} \cos \alpha\left(\lambda+3 \kappa \sin ^{2} \alpha\right)\right] .
\end{aligned}
$$

Trilinear Higgs coupling of the SM-like Higgs $g_{h h h}$ is a very crucial indirect probe of new physics at the current and future colliders. Any deviation from its corresponding SM value,

$$
g_{h h h}^{\mathrm{SM}}=\frac{3 m_{h}^{2}}{v},
$$

would be a signal of new physics. At the high-luminosity LHC (HL-LHC), i.e. $\sqrt{s}=14 \mathrm{TeV}$ with $3000 \mathrm{fb}^{-1}$, it is expected to achieve sensitivity of $\left|1-g_{h h h} / g_{h h h}^{\mathrm{SM}}\right| \sim 0.5$, [44]. However, as we see in the following sections, in most of $\mathrm{TH}$ parameter space trilinear Higgs coupling is very mildly constraining. On the other hand, the triple Higgs coupling $g_{\hat{h} h h}$ is crucial for the decay of heavy twin Higgs to a pair of SM Higgs.

\subsection{Physical basis for Twin Higgs models}

Twin Higgs effective scalar potential (2.6) contains five real parameters, $f_{0}, \lambda, \kappa, \sigma$ and $\rho$. For phenomenological analysis, it is more convenient to choose a basis where these parameters can be expressed in more physical observables. Physical observables for linear TH models are the SM Higgs mass $m_{h}$, heavy twin Higgs mass $m_{\hat{h}}$, SM vev $v$, and twin vev $\hat{v}$ (or equivalently $f$ ). We fix the SM Higgs mass $m_{h}=125 \mathrm{GeV}$ and its vev $v=246 \mathrm{GeV}$. 
This leaves us with three free parameters $m_{\hat{h}}, f$, and hard breaking parameter $\rho$. Since we require $\rho \leq \lambda$, therefore, it is instructive to define $\tilde{\rho}$ as

$$
\tilde{\rho} \equiv \rho / \lambda, \quad \text { such that }|\tilde{\rho}| \lesssim 1
$$

In the following sections we work in $\left(m_{\hat{h}}-f / v\right)$ plane and constraint the parameter space through direct searches of the heavy twin Higgs and indirect limits from the SM Higgs data at the LHC. The trade of potential parameters in the 'physical TH basis' is as,

$$
\underbrace{f_{0}, \lambda, \kappa, \sigma, \rho}_{\text {TH gauge basis }} \Longleftrightarrow \underbrace{v, f, m_{h}, m_{\hat{h}}, \tilde{\rho}}_{\text {TH physical basis }} .
$$

Advantage of the above parametrization is that it leaves the hard breaking parameter $\tilde{\rho}$ as a free parameter, which gives us a handle on hard breaking effects in the model. Potential parameters have the following values in the physical basis:

$$
\begin{aligned}
f_{0} & =f \sqrt{1+\frac{\kappa}{\lambda}\left(1-\xi^{2}\right)}, \quad \sigma=\frac{\lambda\left(\lambda \tilde{\rho} \xi^{2}-\kappa\left(1-2 \xi^{2}\right)\right)}{\lambda+\kappa\left(1-\xi^{2}\right)}, \\
\lambda & =\frac{\left(m_{\hat{h}}^{2}+m_{h}^{2}\right) \tilde{\rho}\left(1-2 \xi^{2}\right)}{4 f^{2}\left(1+\tilde{\rho} \xi^{2}\left(1-\xi^{2}\right)\right)}+\frac{\widehat{\Delta}}{4 f^{2} \xi \sqrt{1-\xi^{2}}\left(1+\tilde{\rho} \xi^{2}\left(1-\xi^{2}\right)\right)}, \\
\kappa & =\frac{\left(m_{\hat{h}}^{2}+m_{h}^{2}\right)\left(2+\tilde{\rho}^{2} \xi^{2}-\tilde{\rho}\left(1-2 \xi^{2}\right)\right)}{4 f^{2}\left(1+\tilde{\rho} \xi^{2}\left(1-\xi^{2}\right)\right)}-\frac{\left(1+\tilde{\rho} \xi^{2}\right) \widehat{\Delta}}{4 f^{2} \xi \sqrt{1-\xi^{2}}\left(1+\tilde{\rho} \xi^{2}\left(1-\xi^{2}\right)\right)},
\end{aligned}
$$

where

$$
\widehat{\Delta} \equiv \sqrt{\left[4\left(m_{\hat{h}}^{2}+m_{h}^{2}\right)^{2}+\left(m_{\hat{h}}^{2}-m_{h}^{2}\right)^{2} \tilde{\rho}^{2}\right] \xi^{2}\left(1-\xi^{2}\right)-4 m_{\hat{h}}^{2} m_{h}^{2}} .
$$

In our phenomenological analysis in sections 3 and 4, we require the following conditions on potential parameters in order to satisfy unitarity and perturbativity constraints,

$$
0<\lambda \leq 4 \pi, \quad|\sigma| \leq \lambda, \quad|\kappa| \leq \lambda, \quad|\rho| \leq \lambda, \quad \text { and } \quad f_{0}^{2}>0
$$

along with the SM Higgs mass $m_{h}=125 \mathrm{GeV}$ and its vev $v=246 \mathrm{GeV}$. If any (or more than one) of the above conditions is not met then the parameter space would be called "unphysical".

\subsection{Electroweak precision constraints in Twin Higgs models}

In this subsection, we briefly comment on the constraints in the TH models from the electroweak precision data when the TH symmetry breaking is linearly realized and a relatively light radial mode (twin Higgs) is present in the effective theory. In particular, the electroweak oblique parameters $S$ and $T$ receive infrared contributions - due to the mixing of the SM-like Higgs with the heavy states and the reduced coupling of the SM-like Higgs to the SM gauge bosons - which are proportional to $\left(1-a^{2}\right) \ln \left(\Lambda^{2} / m_{h}^{2}\right)$, where $a \equiv g_{h V V} / g_{h V V}^{\mathrm{SM}}$ and $\Lambda$ is the scale associated with the heavy states [45]. In the linear TH models, $a=g_{h}=\cos \alpha$ (see figure 16) and the cutoff scale $\Lambda$ is the heavy twin Higgs mass, 
i.e. $\Lambda=m_{\hat{h}}$. Hence, the shifts of the oblique parameters $S$ and $T$ due to the SM-like Higgs and heavy twin Higgs in the TH models are [5, 45],

$$
\Delta S \approx \frac{1}{12} \frac{\sin ^{2} \alpha}{\pi} \ln \left(\frac{m_{\hat{h}}^{2}}{m_{h}^{2}}\right), \quad \Delta T \approx-\frac{3}{16} \frac{\sin ^{2} \alpha}{\pi \cos ^{2} \theta_{W}} \ln \left(\frac{m_{\hat{h}}^{2}}{m_{h}^{2}}\right) .
$$

In figure 2, we employ Gfitter values for $S=0.05 \pm 0.11$ and $T=0.09 \pm 0.13$ with free $U$ for the SM Higgs $m_{h}=125 \mathrm{GeV}$ [46] to construct $68 \%$ (red) and $95 \%$ (lightred) C.L. $S-T$ contours. Twin Higgs contributions to the oblique parameters are shown for $m_{\hat{h}}=(750,1500,2500) \mathrm{GeV}$ as $(+, \times, \diamond)$, respectively, for the color-coded values of $f / v=3$ (blue), 5 (green), 8 (purple). The contribution of a pure SM Higgs of mass $m_{h}=125 \mathrm{GeV}$ is shown as a black star $\star$. The left (right) plot considers hard breaking contributions $\tilde{\rho}=0(0.5)$ in the TH effective potential. Note that the presence of hard breaking contributions $\tilde{\rho}>0(\tilde{\rho}<0)$ slightly worsens (improves) the electroweak precision constraints as compared to the $\tilde{\rho}=0$ case. This is mainly due to the fact that the hard breaking effects are proportional to the mixing angle $\alpha$, see also below. There are two interesting features we observe from figure 2: $(i)$ for a fixed value of $f / v$, the $\mathrm{TH}$ contributions to the oblique parameters $S, T$ are smaller for lighter twin Higgs, and (ii) for a fixed twin Higgs mass, the oblique parameters $S, T$ receive contribution proportional to $v^{2} / f^{2}$, this feature is similar to composite Higgs models. In the following sections for the phenomenological analysis, we implement the electroweak precision constraints at $95 \%$ C.L. on the TH parameter space.

\section{Heavy Higgs phenomenology in Mirror Twin Higgs}

The most general effective renormalizable Lagrangian for the MTH model is ${ }^{4}$

$$
\mathcal{L}_{\mathrm{MTH}}=\mathcal{L}_{321}+\hat{\mathcal{L}}_{\hat{3} \hat{2} \hat{1}}-V_{\text {eff }}(H, \widehat{H}),
$$

where $\mathcal{L}_{321}$ represents full SM Lagrangian with $\mathrm{SU}(3)_{c} \times \mathrm{SU}(2)_{L} \times \mathrm{U}(1)_{Y}$ gauge group excluding the SM Higgs potential and $\hat{\mathcal{L}}_{\hat{3} \hat{2} \hat{1}}$ is the corresponding twin sector Lagrangian. The effective scalar potential $V_{\text {eff }}(H, \widehat{H})$ for the MTH model is given in eq. (2.6). Notable features of the MTH model are:

$\diamond$ Gauge structure of twin Lagrangian $\hat{\mathcal{L}}_{\hat{3} \hat{2} \hat{1}}$ is a mirror copy of the SM Lagrangian $\mathcal{L}_{321}$, and the twin sector particle content is the same as that of the SM.

$\diamond$ Twin sector gauge and Yukawa couplings are almost equal to that of the SM, i.e.

$$
\hat{g}_{s} \simeq g_{s}, \quad \hat{g} \simeq g, \quad \hat{g}^{\prime} \simeq g^{\prime}, \quad \hat{y}_{\hat{f}} \simeq y_{f},
$$

where $g_{s}, g$ and $g^{\prime}$ denote the $\mathrm{SU}(3)_{c}, \mathrm{SU}(2)_{L}$ and $\mathrm{U}(1)_{Y}$ gauge couplings, respectively, whereas $y_{f}$ are the fermion Yukawa couplings. This is required to cancel the quantum corrections to the SM Higgs mass.

\footnotetext{
${ }^{4}$ We have not included a renormalizable $\mathbb{Z}_{2}$ soft breaking term involving mixing of the SM hypercharge $\mathrm{U}(1)_{Y}$ and twin hypercharge $\mathrm{U}(1)_{\hat{Y}}$, i.e. $\varepsilon B_{\mu \nu} \hat{B}^{\mu \nu}$. It provides a vector portal between the $\mathrm{SM}$ and twin sectors, see [47].
} 

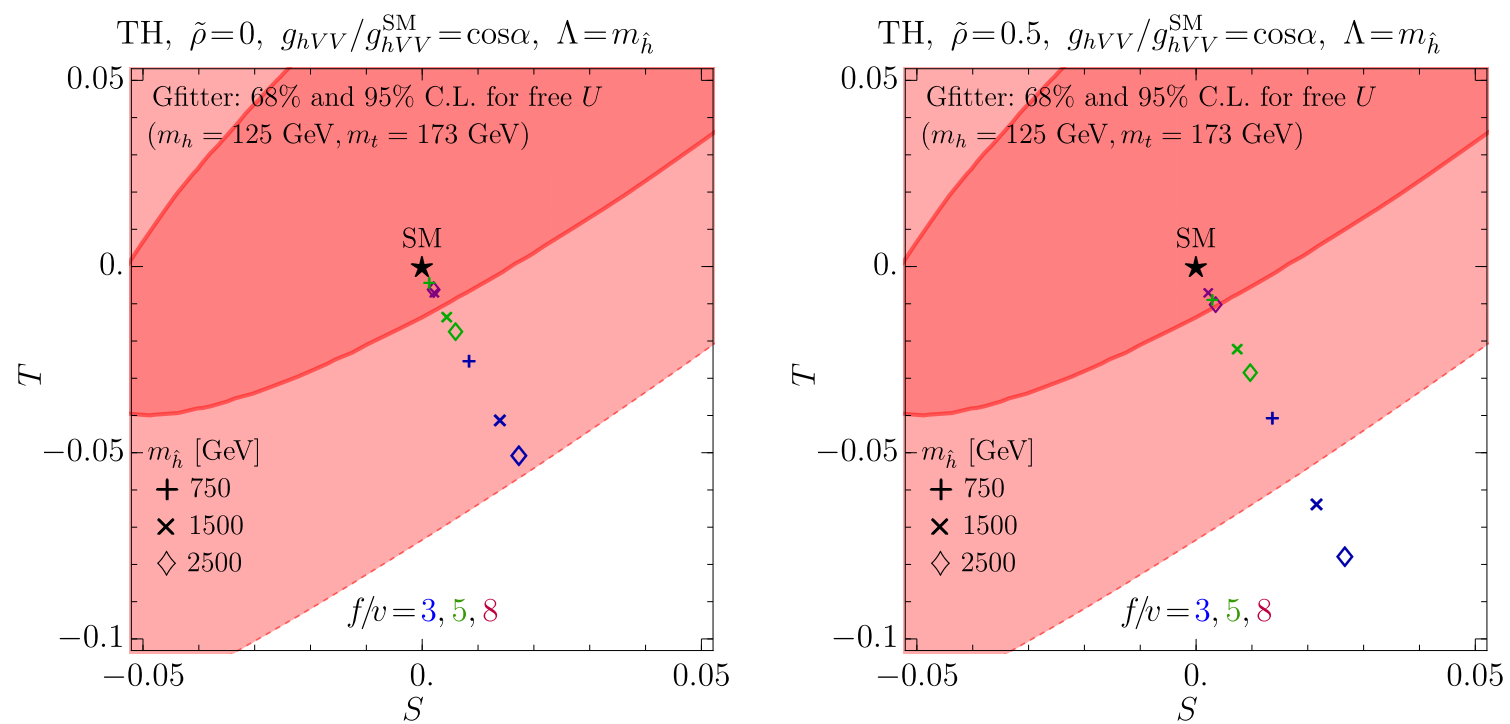

Figure 2. Magnified $68 \%$ (red) and $95 \%$ (light-red) C.L. contours of the oblique parameters $S, T$ showing contributions of the scalar sector of the TH models for $\tilde{\rho}=0$ (left) and $\tilde{\rho}=0$ (right). We consider the twin Higgs mass $m_{\hat{h}}=(750,1500,2500) \mathrm{GeV}$ denoted as $(+, \times, \diamond)$, respectively, for the color-coded values of $f / v=3$ (blue), 5 (green), 8 (purple).

$\diamond$ Masses of the twin sector gauge bosons $m_{\hat{V}}$ and fermions $m_{\hat{f}}$ scale universally w.r.t. the SM counterparts as,

$$
m_{\hat{V}}=\frac{\hat{v}}{v} m_{V}, \quad m_{\hat{f}}=\frac{\hat{v}}{v} m_{f},
$$

where $V=\left(W^{ \pm}, Z\right)$ and $f$ denotes the SM fermions (twin sector are with a hat).

$\diamond$ We assume a mechanism for twin neutrino mass generation exits, see e.g. [32, 48]. However, neutrinos play almost no role in the phenomenology of TH scalar sector so they are irrelevant to our discussion.

We collect the most relevant Feynman rules for the scalar sector of the MTH model in figure 16 (appendix A), where apart from tree-level vertices for the SM and twin-sector massive gauge bosons and fermions, we also include loop induced vertices for massless gauge bosons of the two sectors. Note that the SM-like Higgs couplings to the SM sector particles are suppressed by $g_{h}=\cos \alpha$ to their respective SM values, which is a common feature of BSM scenarios where the SM Higgs mixes with another scalar with a mixing angle $\alpha$. The SM Higgs coupling measurements at the LHC put severe constraints on the mixing angle and exclude some of the parameter space of the MTH model.

We collect formulae for partial decay widths of heavy twin Higgs to the SM and twin states in appendix A. For computing heavy twin Higgs cross-sections, we adopt a strategy similar to the one outlined in [49]. For different initial (production) and final (decay) states, the cross-section of heavy twin Higgs is parametrized as:

$$
\sigma_{\hat{h} \rightarrow X X}^{Y Y} \equiv \sigma(Y Y \rightarrow \hat{h}) \cdot \mathcal{B}(\hat{h} \rightarrow X X)=\left.\sigma^{\mathrm{SM}}(Y Y \rightarrow h)\right|_{m_{h}=m_{\hat{h}}} \mathcal{C}_{\hat{h} Y Y}^{2} \mathcal{B}(\hat{h} \rightarrow X X),
$$


where $\sigma^{\mathrm{SM}}(Y Y \rightarrow h)$ is the SM-like Higgs production cross-section calculated at the heavy twin Higgs mass for $Y Y=(g g, V V)$. The SM Higgs cross-sections $\left.\sigma^{\mathrm{SM}}(h \rightarrow Y Y)\right|_{m_{h}=m_{\hat{h}}}$ are obtained from Higgs Cross-section Working Group [50]. Above $\mathcal{B}(\hat{h} \rightarrow X X)$ is the twin Higgs branching fraction to $X X$ final state, whereas, effective coupling $\mathcal{C}_{\hat{h} Y Y}$ is defined as,

$$
\mathcal{C}_{\hat{h} Y Y}^{2} \equiv \frac{\sigma(Y Y \rightarrow \hat{h})}{\left.\sigma^{\mathrm{SM}}(Y Y \rightarrow h)\right|_{m_{h}=m_{\hat{h}}}}=\frac{\Gamma(\hat{h} \rightarrow Y Y)}{\left.\Gamma^{\mathrm{SM}}(h \rightarrow Y Y)\right|_{m_{h}=m_{\hat{h}}}},
$$

where $\left.\Gamma^{\mathrm{SM}}(h \rightarrow Y Y)\right|_{m_{h}=m_{\hat{h}}}$ is the SM Higgs partial decay width evaluated at the heavy Higgs mass and $\Gamma(\hat{h} \rightarrow Y Y)$ is the twin Higgs decay width, see appendix A. For $Y Y=g g$ and $V V$, one finds the effective twin Higgs coupling,

$$
\mathcal{C}_{\hat{h} g g}^{2}=\mathcal{C}_{\hat{h} V V}^{2}=\left|g_{\hat{h}}\right|^{2}=\sin ^{2} \alpha,
$$

which is a universal rescaling of heavy twin Higgs production at the LHC and future colliders. Note that the main production channel for the heavy twin Higgs is gluon-gluon fusion $(g g \mathrm{~F})$, whereas, the vector-boson fusion ( $\mathrm{VBF})$ is subdominant in most of the parameter space of TH models. Similarly, for the SM Higgs with $m_{h}=125 \mathrm{GeV}$, the cross-section times branching fractions are given as,

$$
\sigma_{h \rightarrow X X}^{Y Y} \equiv \sigma(Y Y \rightarrow h) \cdot \mathcal{B}(h \rightarrow X X)=\sigma^{\mathrm{SM}}(Y Y \rightarrow h) \mathcal{C}_{h Y Y}^{2} \mathcal{B}(h \rightarrow X X),
$$

where for $Y Y=(g g, V V)$ the effective SM Higgs coupling is

$$
\mathcal{C}_{h g g}^{2}=\mathcal{C}_{h V V}^{2}=\left|g_{h}\right|^{2}=\cos ^{2} \alpha .
$$

It is convenient to define SM Higgs signal-strength $\mu_{h \rightarrow X X}^{Y Y}$ as,

$$
\mu_{h \rightarrow X X}^{Y Y} \equiv \frac{\sigma(Y Y \rightarrow h) \cdot \mathcal{B}(h \rightarrow X X)}{\sigma^{\mathrm{SM}}(Y Y \rightarrow h) \cdot \mathcal{B}^{\mathrm{SM}}(h \rightarrow X X)}=\mathcal{C}_{h Y Y}^{2} \frac{\mathcal{B}(h \rightarrow X X)}{\mathcal{B}^{\mathrm{SM}}(h \rightarrow X X)}
$$

The SM Higgs signal-strength measurements in many production and decay channels have been measured with unforeseeable precision at the LHC run-1 [51] and they provide stringent constraints on BSM models.

For numerical analysis of the twin Higgs phenomenology, we employ a modified version of HDECAY code [52] incorporating the twin sector, where all the appropriate QCD and electroweak corrections are included in the SM as well as in the twin sector. Note that in the MTH running of twin gauge couplings is similar to those of the SM. We also assume that at the cutoff scale $\Lambda$, the SM and twin gauge coupling as well the Yukawa couplings are exactly equal. Moreover, the heavy twin Higgs (and the SM-like Higgs) decays to the twin states are invisible at the colliders.

In figure 3 we show branching fractions (larger than a percent) of heavy twin Higgs for different final states of the SM and twin (invisible) sector as a function of the heavy Higgs mass for $f / v=3$ (left-panel) and 6 (right-panel), and $\tilde{\rho}=0$. Notice the lower mass of the heavy Higgs is shifted to higher values as $f / v$ increases. This is due to the fact that with the increase in $f / v$ the lower twin Higgs masses do not reconcile with the SM Higgs 

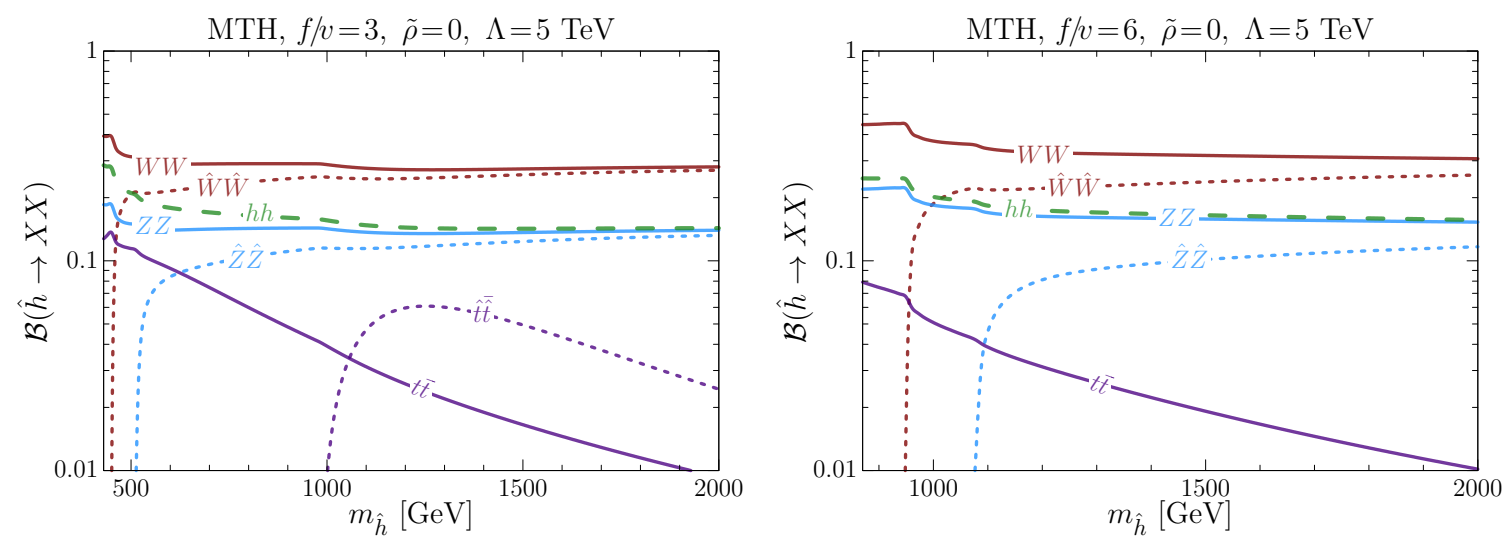

Figure 3. These plots show heavy twin Higgs branching ratios in the MTH to different final states as a function of its mass. We take $f / v=3$ (left-panel) and 6 (right-panel) for $\tilde{\rho}=0$ and $\Lambda=5 \mathrm{TeV}$.

mass $125 \mathrm{GeV}$. For a given production mode (e.g. gluon fusion), the relative magnitudes of these branching ratios determine the relative rates for the various final states. In the absence of the $\mathbb{Z}_{2} / \mathrm{SU}(4)$ hard breaking term, i.e. $\tilde{\rho}=0$, it is clear from figure 3 the heavy Higgs branching fractions to the SM vector bosons $W^{ \pm}, Z$, Higgs $h$, and the twin vector bosons $\hat{W}^{ \pm}, \hat{Z}$, are the most dominant channels. Moreover, for $\tilde{\rho}=0$ we find,

$$
\mathcal{B}(\hat{h} \rightarrow h h) \simeq \mathcal{B}(\hat{h} \rightarrow Z Z) \simeq \frac{1}{2} \mathcal{B}(\hat{h} \rightarrow W W) \simeq \mathcal{B}(\hat{h} \rightarrow \hat{Z} \hat{Z}) \simeq \frac{1}{2} \mathcal{B}(\hat{h} \rightarrow \hat{W} \hat{W}),
$$

which is a clear manifestation of the Goldstone boson equivalence theorem. Since the longitudinal modes of massive vector bosons and the SM Higgs are Goldstone modes of the TH spontaneous symmetry breaking. Since the radial mode couples universally to all the Goldstone modes, therefore, one would expect that the branching ratio of heavy twin Higgs in the visible $\left(W^{ \pm}, Z, h\right)$ sector would be $\sim 4 / 7$ and that in the invisible $\left(\hat{W}^{ \pm}, \hat{Z}\right)$ sector would be $\sim 3 / 7$, see also [36]. This gives a profound prediction for the TH models that the decay rates of heavy Higgs to the SM Higgs and vector bosons (say $Z Z$ ) are equal. Such an observation at the LHC and/or future colliders would provide a clear distinction between TH and other BSM models.

On the other hand, in the presence of explicit $\mathbb{Z}_{2} / \mathrm{SU}(4)$ hard breaking $\rho$-term in the TH scalar potential (2.6), the above-mentioned universality among the interactions of heavy twin Higgs to the SM Higgs/vector bosons and twin vector bosons would break. To see this explicit symmetry breaking effects, in figure 4 we plot the twin Higgs branching fractions for $\tilde{\rho}=0.5$ and -0.5 , where $\tilde{\rho} \equiv \rho / \lambda$. As we are working in EFT framework where the sign and/or the size of explicit hard braking term is a model-dependent quantity so we consider both sign possibilities, see for instance [16]. Notice for the case of positive $\tilde{\rho}$ (figure 4 left-panel), there is a sharp increase of SM branching fractions, whereas this behavior is reversed for the case of negative $\tilde{\rho}$ (right-panel), as compared to figure 3 (leftpanel) where $\tilde{\rho}=0$. The reason for this is the effects of explicit breaking on mixing angle $\alpha$, which increases for positive $\tilde{\rho}$ and vice versa (see also (2.16)). Hence, $\tilde{\rho}>0$ enhances the rates of heavy twin Higgs to the SM sector, whereas $\tilde{\rho}<0$ suppresses those rates. As 

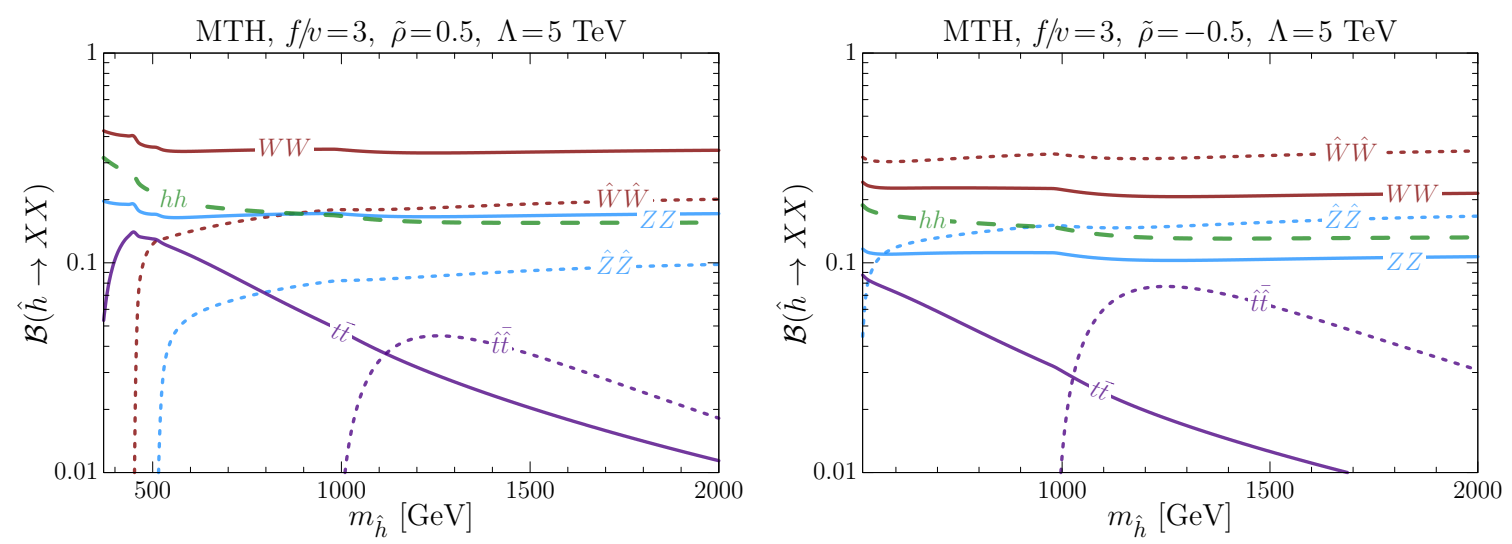

Figure 4. Same as figure 3 but for explicit hard breaking parameter $\tilde{\rho}=0.5$ (left) and -0.5 (right).

a consequence, the LHC (and/or future colliders) would be able to probe more region of TH parameter space with $\tilde{\rho}>0$ than for $\tilde{\rho}<0$ (see below).

In figure 5 we show parameter space of the MTH model in $m_{\hat{h}}-f / v$ plane with contours of heavy twin Higgs cross-sections to a pair of SM vector bosons i.e. $\sigma_{\hat{h} \rightarrow Z Z}^{g g \mathrm{~F}} \equiv$ $\sigma(g g \rightarrow \hat{h}) \cdot \mathcal{B}(\hat{h} \rightarrow Z Z)$ (solid blue curves), and SM Higgs signal-strength $\mu_{h \rightarrow Z Z}^{g g \mathrm{~F}}$ (dashed red curves). The cross-section is calculated at the LHC with the center-of-mass energy $14 \mathrm{TeV}$. The left and right plots in figure 5 are for hard breaking parameter $\tilde{\rho}=0$ and $\tilde{\rho}=0.5$, respectively. Similarly, figure 6 presents contours of heavy twin Higgs cross-section to a pair of SM Higgs bosons, $\sigma_{\hat{h} \rightarrow h h}^{g g \mathrm{~F}} \equiv \sigma(g g \rightarrow \hat{h}) \cdot \mathcal{B}(\hat{h} \rightarrow h h)$ (solid green curves). The description of shaded regions in figures 5 and 6 are as follows:

- Gray region of figures 5 and 6 represents unphysical parameter space excluded by the theoretical constraints as outlined in the previous section.

- Orange area (figures 5 and 6) captures parameter spaces where heavy twin Higgs width is greater than its mass, $\Gamma_{\hat{h}}>m_{\hat{h}}$.

- Red shaded region (figures 5 and 6 ) is excluded at 95\% C.L. by the combined ATLAS and CMS analysis of the SM Higgs signal-strength $\left(\mu_{h \rightarrow Z Z}^{g g \mathrm{~F}}<0.79\right)$ at the LHC [51].

- Purple area (figure 5) shows 95\% C.L. exclusion by direct searches of heavy scalar decaying to the SM $V V$ final state at the ALTAS experiment with $13 \mathrm{TeV}$ [53-55].

- Blue region (figure 5) presents projected 95\% C.L. exclusion limit for cross-section times branching fraction of heavy twin Higgs to SM vector bosons $\sigma_{\hat{h} \rightarrow Z Z}^{g g \mathrm{~F}}$ for the HLLHC at $14 \mathrm{TeV}$ with an integrated luminosity of $3000 \mathrm{fb}^{-1}$. This estimate is adopted from [36].

- Green shaded area (figure 6) shows projected 95\% C.L. exclusion limit for crosssection times branching fraction of heavy Higgs to a pair of SM Higgs bosons $\sigma_{\hat{h} \rightarrow h h}^{g g \mathrm{~F}}$ at the HL-LHC, as adopted from [36]. 

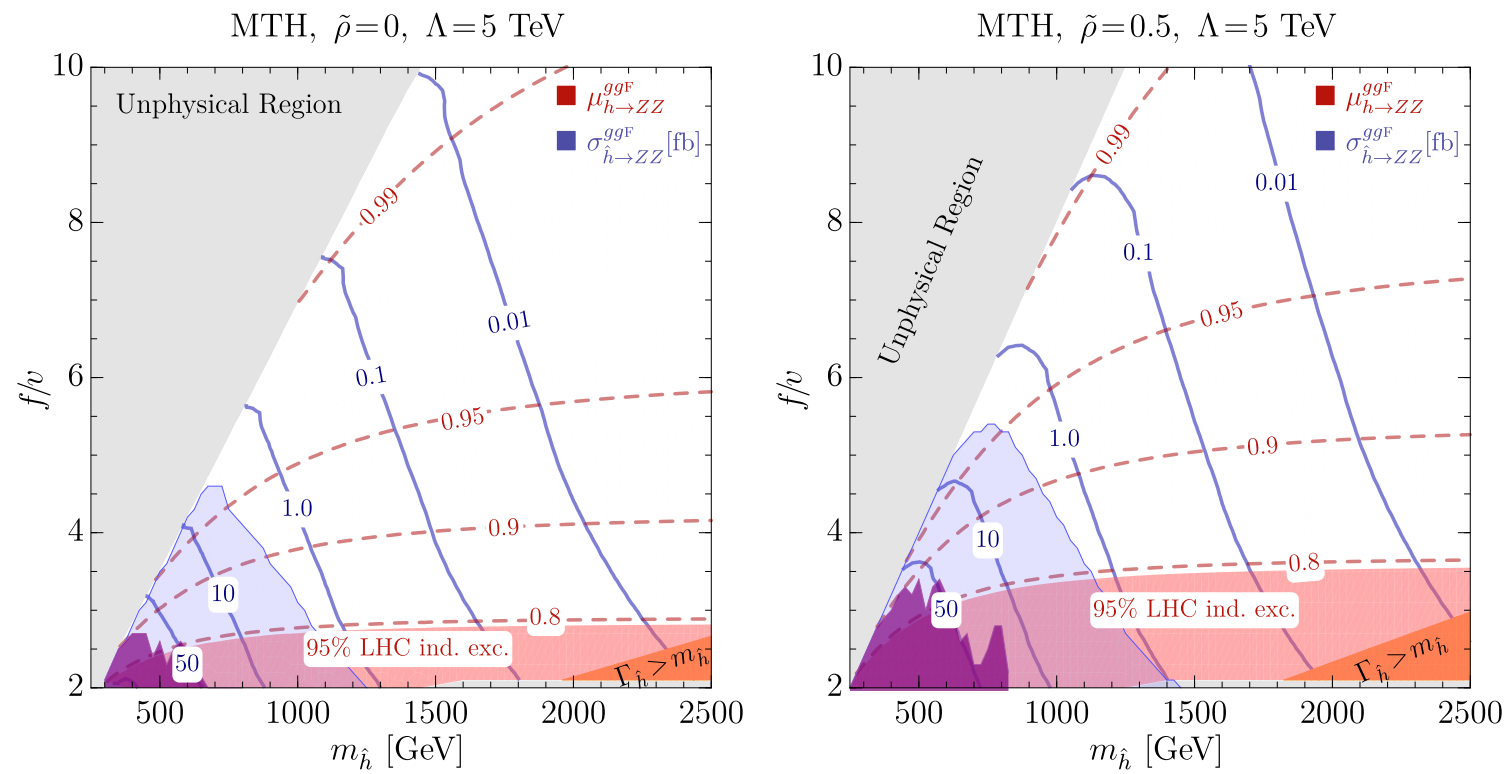

Figure 5. These plots show parameter space in $m_{\hat{h}}-f / v$ plane for the MTH model. The contours of heavy twin Higgs cross-section to the SM $Z$-boson $\sigma_{\hat{h} \rightarrow Z Z}^{g g \mathrm{~F}}[\mathrm{fb}]$ (solid blue) and SM Higgs signalstrength $\mu_{h \rightarrow Z Z}^{g g \mathrm{~F}}$ (dashed red) are shown for $\tilde{\rho}=0$ (left) and $\tilde{\rho}=0.5$ (right). Shaded regions read as: unphysical parameters (gray), $\Gamma_{\hat{h}}>m_{\hat{h}}$ (orange), exclusion by the current LHC direct searches $\hat{h} \rightarrow Z Z$ (purple), the projected reach for direct searches $\hat{h} \rightarrow Z Z$ at the HL-LHC (light-blue), and exclusion by the SM Higgs signal-strength measurements at the LHC run-1 (red).
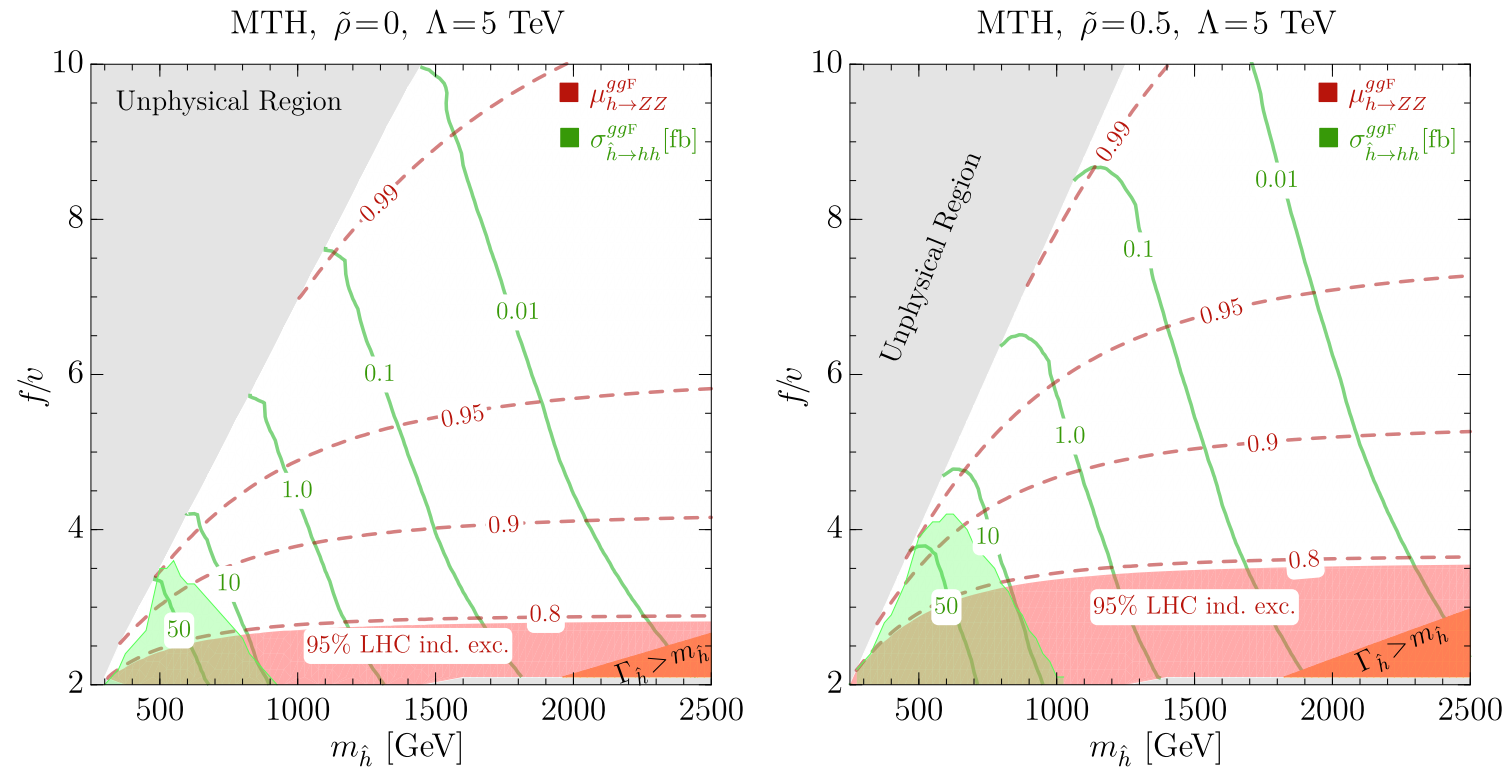

Figure 6. These plots show parameter space in $m_{\hat{h}}-f / v$ plane for the MTH model. The contours of heavy twin Higgs cross-section to a pair SM Higgs bosons $\sigma_{\hat{h} \rightarrow h h}^{g g \mathrm{~F}}[\mathrm{fb}]$ (solid green) and SM Higgs signal-strength $\mu_{h \rightarrow Z Z}^{g g \mathrm{~F}}$ (dashed red) are shown for $\tilde{\rho}=0$ (left) and $\tilde{\rho}=0.5$ (right). Shaded regions read as: unphysical parameters (gray), $\Gamma_{\hat{h}}>m_{\hat{h}}$ (orange), the projected reach for direct searches $\hat{h} \rightarrow h h$ at the HL-LHC (light-green), and exclusion by the SM Higgs signal-strength measurements at the LHC run-1 (red). 
From figures 5 and 6 one can notice a clear enhancement in the heavy twin Higgs cross-sections to the SM vector bosons and the Higgs boson for $\tilde{\rho}=0.5$ as compared to $\tilde{\rho}=0$ case. As we discussed above, by turning on the hard breaking effects $(\tilde{\rho}>0)$ not only cross-sections of heavy twin Higgs to the visible sector would increase due to increase in the mixing angle but also the SM Higgs signal-strength would increase. As a result, on one hand, the observability potential of heavy twin Higgs through direct search would increase at colliders, on the other hand, the indirect constraints from the SM Higgs signalstrength measurements would become stringent. However, we note that for $\tilde{\rho}<0$ the mixing angle decreases, hence making it harder to exclude the parameter space via direct or indirect searches at colliders. As discussed above, a generic prediction of the TH models is that heavy Higgs branching fractions to $h h$ is comparable to that of the $V V$, hence the signal of heavy Higgs to a pair of SM Higgs is very important for the discovery potential of TH mechanism. However notice that the HL-LHC reach for $\sigma_{\hat{h} \rightarrow h h}^{g g \mathrm{~F}}$ is about an order of magnitude less than that of the $\sigma_{\hat{h} \rightarrow Z Z}^{g g \mathrm{~F}}$. We summarize results for the MTH parameter scan in figure 5 and 6 as:

(i) The LHC run-1 and early run-2 data only constraints a small region of parameters $f / v \lesssim 3$ for heavy twin Higgs decays to the SM $V V$.

(ii) Projected reach of direct searches at the HL-LHC indicates that the MTH model with $m_{\hat{h}} \lesssim 1 \mathrm{TeV}$ and $f / v \lesssim 5$ would be accessible.

(iii) Direct searches are more sensitive to the low mass region, whereas the indirect constraints are more effective in the high mass regions of the MTH model. Current indirect constraint implies $f / v \lesssim 3$, however the reach for the signal-strength measurement at the HL-LHC is about 5\% [44] which implies constraints up to $f / v \lesssim 6$.

For completeness in figure 7 we show the contours of the ratio of heavy twin Higgs width over its mass $\Gamma_{\hat{h}} / m_{\hat{h}}$ (solid orange), the ratio of the SM-like Higgs trilinear coupling over its SM value $g_{h h h} / g_{h h h}^{\mathrm{SM}}$ (dashed purple), and the SM Higgs invisible (twin) branching fraction $\mathcal{B}\left(h \rightarrow\right.$ inv.) (dotted), in $m_{\hat{h}}-f / v$ plane for $\tilde{\rho}=0$ (left) and $\tilde{\rho}=0.5$ (right). Moreover, in figure 7 we also show $95 \%$ C.L. constraints on the TH parameter space due to the EWPT (gray hatched region), see section 2.2 for details. Note the indirect probes via the SM-like Higgs trilinear coupling $g_{h h h} / g_{h h h}^{\mathrm{SM}}$ and/or the SM Higgs invisible (twin) branching fraction $\mathcal{B}(h \rightarrow$ inv $)$ are far less constraining at the $\mathrm{LHC}^{5}$ than the Higgs coupling measurements. However, at the future colliders they can be complimentary probe channels.

\section{Heavy Higgs phenomenology in Fraternal Twin Higgs}

The FTH model has the minimal twin sector matter content required to cancel the radiative corrections to the SM Higgs mass [5]. The Lagrangian for the FTH model is,

$$
\mathcal{L}_{\mathrm{FTH}}=\mathcal{L}_{321}+\hat{\mathcal{L}}_{\hat{3} \hat{2}}-V_{\text {eff }}(H, \widehat{H}),
$$

\footnotetext{
${ }^{5}$ The most stringent current bound on the SM Higgs invisible decays $\mathcal{B}(h \rightarrow$ inv. $) \leq 0.24$ is by CMS [56].
} 

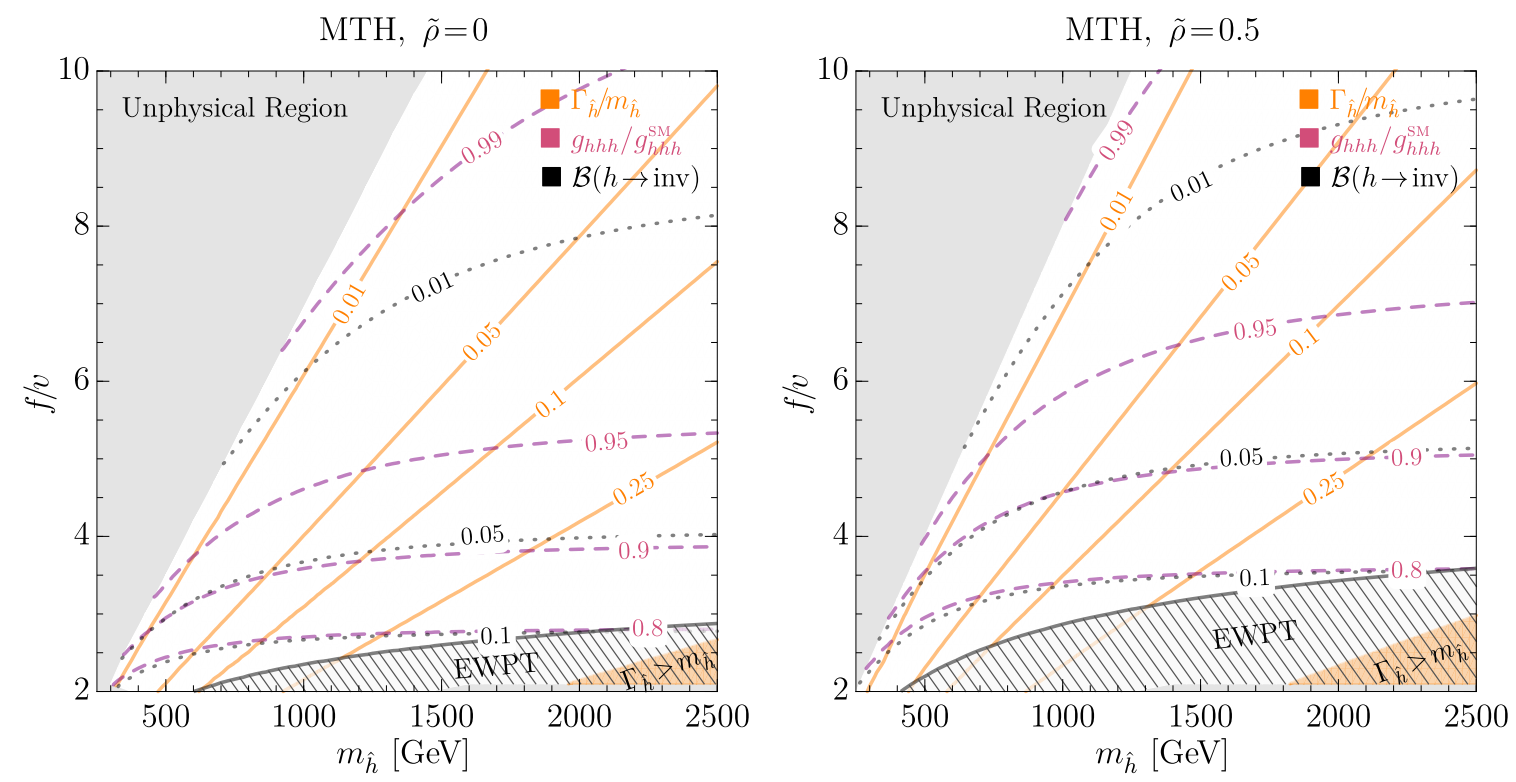

Figure 7. These plots show parameter space in $m_{\hat{h}}-f / v$ plane for the MTH model with $\tilde{\rho}=0$ (left) and $\tilde{\rho}=0.5$ (right). The contours of the ratio of heavy twin Higgs width over its mass $\Gamma_{\hat{h}} / m_{\hat{h}}$ (solid orange), the ratio of the SM-like Higgs trilinear coupling over its corresponding SM values $g_{h h h} / g_{h h h}^{\mathrm{SM}}$ (dashed purple), and the SM Higgs invisible (twin) branching fraction $\mathcal{B}(h \rightarrow$ inv.) (dotted). The gray area corresponds to the unphysical parameters, the hatched-gray region is excluded by the EWPT at $95 \%$ C.L., and the orange region represents $\Gamma_{\hat{h}}>m_{\hat{h}}$.

where $\mathcal{L}_{321}$ is the SM Lagrangian and $V_{\text {eff }}(H, \widehat{H})$ is the scalar potential (same as the MTH model) given in eq. (2.6). However, the FTH model differs from the MTH case in the twin sector Lagrangian, $\hat{\mathcal{L}}_{\hat{3} \hat{2}}$, especially in the matter content. The most salient features of the FTH model as compared to the MTH case are as follows [5]:

$\diamond$ Gauge symmetry of the FTH Lagrangian $\hat{\mathcal{L}}_{\hat{3} \hat{2}}$ is $\widehat{\mathrm{SU}}(3)_{c} \times \widehat{\mathrm{SU}}(2)_{L}$, i.e. there is no twin hypercharge $\mathrm{U}(1)_{\hat{Y}}$ gauge group and hence, no twin photon in the FTH model.

$\diamond$ Twin fermionic part of the FTH model contains only third generation of twin quarks $(\hat{t}, \hat{b})$ and leptons $\left(\hat{\tau}, \hat{\nu}_{\tau}\right)$, which are necessary to cancel the SM radiative corrections to the SM Higgs mass.

$\diamond$ Twin sector gauge couplings are required to be very close (better than 1\%) to that of the SM at the cutoff scale $\Lambda$, i.e.

$$
\hat{g}_{s}(\Lambda) \simeq g_{s}(\Lambda), \quad \hat{g}(\Lambda) \simeq g(\Lambda) .
$$

Similarly, twin top Yukawa coupling $\hat{y}_{\hat{t}}$ is required to be very close to the SM top Yukawa coupling, i.e. $\hat{y}_{\hat{t}} \simeq y_{t}$. These requirements are motivated by the naturalness arguments in cancellation of the radiative corrections.

$\diamond$ Contrary to the top Yukawa coupling, the light twin fermions Yukawa couplings $\left(\hat{y}_{\hat{b}}, \hat{y}_{\hat{\tau}}\right)$ could be substantially different from their SM counterparts. 
$\diamond$ Twin sector masses of the weak gauge bosons $m_{\hat{V}}$ and fermions $m_{\hat{f}}$ scale w.r.t. their SM counterparts as,

$$
m_{\hat{V}}=\frac{\hat{v}}{v} m_{V}, \quad m_{\hat{f}}=\frac{\hat{v}}{v} \frac{\hat{y}_{\hat{f}}}{y_{f}} m_{f},
$$

where the light twin fermions receive an extra $\hat{y}_{\hat{f}} / y_{f}$ enhancement in their masses (and also in their couplings) as compared to the MTH model.

$\diamond$ The light twin fermions Yukawa couplings $\left(\hat{y}_{\hat{b}}, \hat{y}_{\hat{\tau}}\right)$ are essentially free parameters. Which implies an enhancement of the heavy Higgs (and the SM Higgs if $m_{\hat{f}}<m_{h} / 2$ ) decay rates to light twin fermions by a factor $y_{\hat{f}}^{2} / y_{f}^{2}$ as compared to the MTH case.

$\diamond$ The most remarkable feature of the FTH model is the relative large QCD confinement scale (due to faster running of the twin QCD coupling constant $\hat{\alpha}_{\mathrm{QCD}}$ ) and subsequent hadronization of twin gluons/bottom quarks to twin glueball/bottomonium states, respectively. In particular, the $0^{++}$twin hadrons mix with the Higgs bosons and lead to prompt and/ or displaced exotic decays to the SM light fermions.

In the FTH model, the heavy twin Higgs decay rates and cross-sections to the SM sector would be almost same as for the MTH case (section 3). Moreover, the twin weak gauge bosons $\hat{W}, \hat{Z}$ and twin top $\hat{t}$ of the FTH have the same masses and coupling to heavy twin Higgs as those for the MTH case. Therefore, their the branching fractions/cross-sections would not change substantially as compared to the MTH case. However, light twin fermion Yukawa couplings can be enhanced, as a result their production cross-sections would get enhancement. To be precise, heavy twin Higgs decay rates to the light twin fermions get an extra $\left(\hat{y}_{\hat{f}} / y_{f}\right)^{2}$ enhancement factor as compared to that of the MTH model. The on-shell decays of heavy Higgs to a pair of twin massive vectors bosons $(\hat{h} \rightarrow \hat{V} \hat{V})$ remain same as in the MTH scenario. However, the off-shell decays $\hat{h} \rightarrow \hat{V}^{*} \hat{V}^{*} \rightarrow 4 \hat{f}$ involve total widths of the vector bosons $\Gamma_{\hat{V}}$, which change as compared to the MTH case due to fewer light twin fermions. In the FTH model, total decay widths of the twin bosons $\hat{W}$ and $\hat{Z}$ are (assuming $m_{\hat{f}} \ll m_{\hat{V}}$ ),

$$
\Gamma\left(\hat{W} \rightarrow \hat{\tau} \overline{\hat{\nu}}_{\tau}\right)=\frac{\hat{g}^{2} m_{\hat{W}}}{48 \pi}, \quad \Gamma(\hat{Z} \rightarrow \hat{f} \overline{\hat{f}})=\frac{\hat{g}^{2} m_{\hat{Z}}}{48 \pi}\left(\hat{g}_{V}^{2}+\hat{g}_{A}^{2}\right),
$$

where $\hat{g}_{V}$ and $\hat{g}_{A}$ are the vector and axial couplings of the twin fermions $\hat{f}=\left(\hat{b}, \hat{\tau}, \hat{\nu}_{\tau}\right)$.

In figure 8 , we show the heavy twin Higgs branching fractions $(\geq 1 \%)$ to the SM and twin states, where the left- and right-panel are for $y_{\hat{b}} / y_{b} \simeq 1.2$ and 6 , respectively, for fix values of $f / v=3, \tilde{\rho}=0$, and $\hat{\Lambda}_{\mathrm{QCD}}=7.35 \mathrm{GeV}$ (which corresponds to the lightest glueball mass $\left.m_{\hat{0}^{+}}=50 \mathrm{GeV}\right)$. For simplicity, in this work we take twin tau Yukawa as $y_{\hat{\tau}} / y_{\tau}=y_{\hat{b}} / y_{b}$, which fixes the twin sector fermion masses up to only one free parameter, the twin bottom Yukawa coupling $\hat{y}_{\hat{b}}$ (or $m_{\hat{b}}$ ). Notice the heavy twin Higgs branching fractions do not substantially change for the twin massive gauge bosons and twin top for $y_{\hat{b}} / y_{b} \simeq 1.2$ and 6 cases. However, the heavy twin Higgs branching fraction for twin bottom quark, $\mathcal{B}(\hat{h} \rightarrow \hat{b} \hat{b}$ ) (shown as dotted curve in figure 8), increases about an order of magnitude for a given $m_{\hat{h}}$ value as we increase $y_{\hat{b}} / y_{b}$ from $\sim 1$ to 6 . The effect of explicit hard breaking 

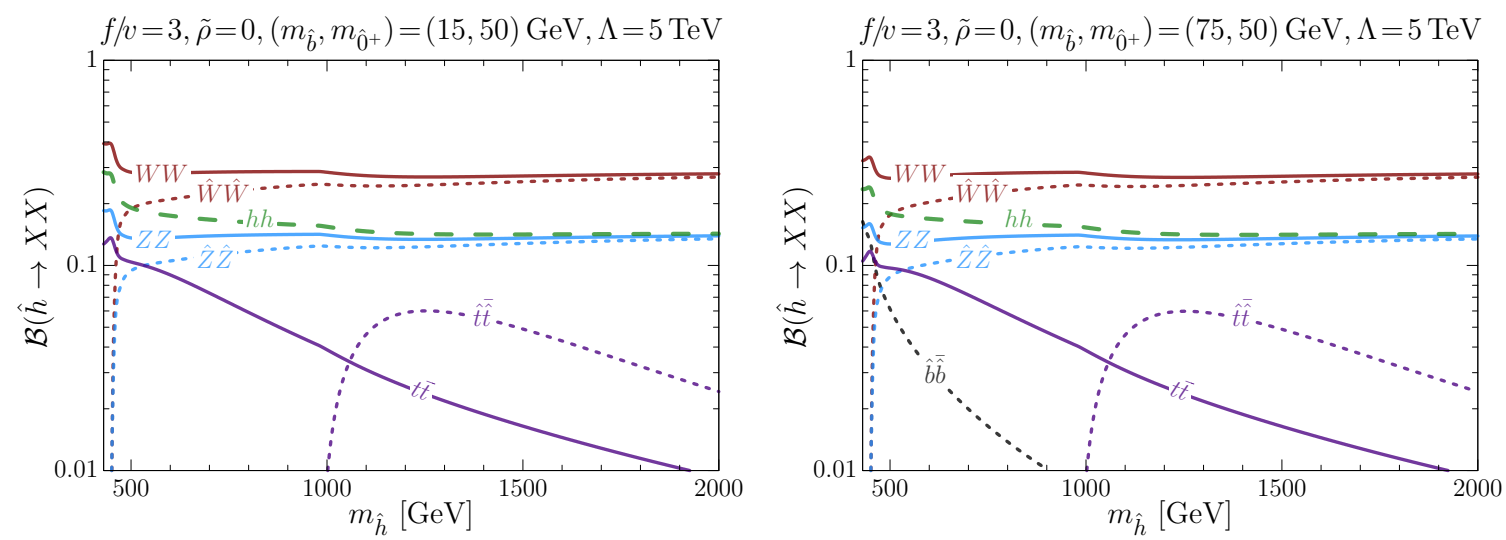

Figure 8. These plots show branching ratios of heavy twin Higgs to different final states as a function its mass in the FTH model. The left and right plots are for $\left(m_{\hat{b}}, m_{\hat{0}^{+}}\right)=(15,50) \mathrm{GeV}$ and $(75,50) \mathrm{GeV}$, respectively, where $f / v=3$ and $\tilde{\rho}=0$.

parameter $\tilde{\rho}$ on the heavy Higgs branching fractions is similar to the MTH case, i.e. there is an enhancement in the SM branching fractions and subsequently suppression to twin sector branching ratios for $\tilde{\rho}>0$, and vice versa for $\tilde{\rho}<0$.

The most notable difference of the FTH model as compared to the MTH case, is the running of twin $\mathrm{QCD}$ coupling constant $\hat{\alpha}_{\mathrm{QCD}}$ from the cutoff scale $\Lambda$ to the confinement scale $\hat{\Lambda}_{\mathrm{QCD}}$. Since the number of quark flavors is less in the FTH (so faster running) as a result the confinement scale $\hat{\Lambda}_{\mathrm{QCD}}$ would be larger than the SM QCD confinement scale. In the following numerical analysis we employ the NNLO twin QCD corrected $\hat{\alpha}_{\mathrm{QCD}}$, however, we require at the cutoff scale $\Lambda$,

$$
0.75 \lesssim \frac{\hat{\alpha}_{\mathrm{QCD}}(\Lambda)}{\alpha_{\mathrm{QCD}}(\Lambda)} \lesssim 1.25
$$

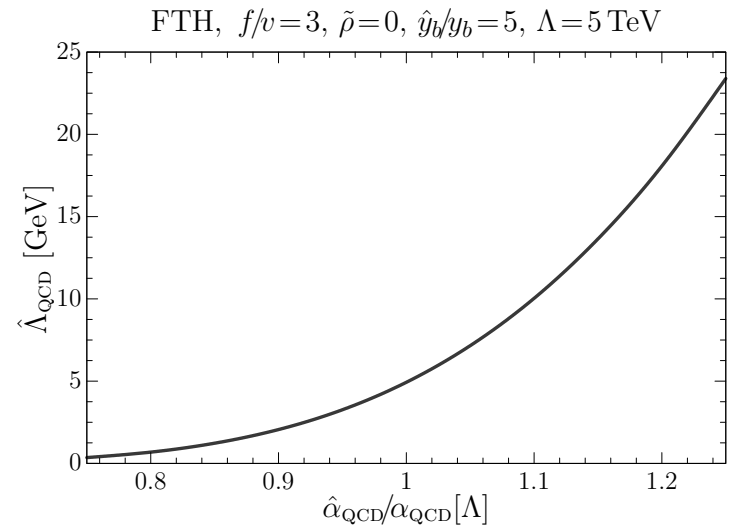

Figure 9. The twin confinement scale $\hat{\Lambda}_{\mathrm{QCD}}$ as a function of $\hat{\alpha}_{\mathrm{QCD}} / \alpha_{\mathrm{QCD}}$ at the cutoff $\Lambda=5 \mathrm{TeV}$.

The above variation in the twin strong coupling constant induce a rather mild fine-tuning $\sim 30 \%$ in the SM Higgs mass. A small variation of $\hat{\alpha}_{\mathrm{QCD}}$ as compared to $\alpha_{\mathrm{QCD}}$ at the high scale $\Lambda$ significantly change the confinement scale which has very crucial phenomenological consequences. In figure 9 we plot the twin confinement scale $\hat{\Lambda}_{\mathrm{QCD}}$ as function of $\hat{\alpha}_{\mathrm{QCD}} / \alpha_{\mathrm{QCD}}$ at the cutoff scale $\Lambda=5 \mathrm{TeV}$, where we kept fix $f / v=3, \tilde{\rho}=0$, and $y_{\hat{b}} / y_{b}=5$.

\subsection{Fraternal Twin hadron production and decays}

In the FTH model due to fewer twin quark flavors and potentially large confinement scale $\hat{\Lambda}_{\mathrm{QCD}}$, the twin QCD confines to form twin glueball and bottomonium $[\hat{b} \bar{b}]$ states. The lightest of the twin glueballs is a $0^{++}$state $\hat{G}_{0^{+}}$with mass $m_{\hat{0}^{+}} \simeq 6.8 \hat{\Lambda}_{\mathrm{QCD}}$. Notice that the lightest glueball $\hat{G}_{0^{+}}$has the correct quantum numbers to mix with the SM Higgs (and 
also twin Higgs) which allows its decay to the SM light fermions. In analogy to the SM bottomonium spectra, the lightest twin bottomonium state is $\hat{\eta}\left(0^{-+}\right)$, and next to lightest is $\hat{\Upsilon}\left(1^{--}\right)$state. However, the $0^{++}$p-wave state $\hat{\chi}_{0}$ with mass $m_{\hat{\chi}} \simeq 2 m_{\hat{b}}$ has the correct quantum numbers to mix with the TH scalar sector such that $\hat{\chi}_{0}$ decays in the visible sector are also possible. Phenomenological aspects of production and decays of the twin glueball and twin bottomonium states due to the SM-like Higgs has been discussed in [5, 39-42], see also [6-11, 57-60]. In this work, we follow these analyses closely but focus on the production of twin hadrons due to heavy twin Higgs.

Since twin hadronization is poorly understood, especially, without the proper knowledge of the non-perturbative twin QCD effects, its hard to make a precise estimate of twin hadron production. However, the perturbative estimates can be made quite accurately and the ignorance of twin hadronization can be controllably parametrized, see for instance [5, 11, 39, 41, 42]. The twin QCD hadronization of twin gluons and bottom quarks lead to a dozen of meta-stable states in their spectrums. However, we are interested in the production of $0^{++}$states, i.e. twin glueball $\hat{G}_{0^{+}}$and twin bottomonium $\hat{\chi}_{0}$, via the scalar sector of the FTH model. Such that the $0^{++}$twin hadrons mix with the SM Higgs and lead to exotic decays to the SM light fermions.

We parametrize heavy twin Higgs partial decay rate to twin glueball $\hat{G}_{0^{+}}$and twin bottomonium $\hat{\chi}_{0}$ states as,

$$
\begin{gathered}
\Gamma\left(\hat{h} \rightarrow \hat{G}_{0^{+}}\right)=\Gamma(\hat{h} \rightarrow \hat{g} \hat{g})\left\langle N_{\hat{G}}\right\rangle \hat{\kappa}_{0} \sqrt{1-\frac{4 m_{\hat{0}^{+}}^{2}}{m_{\hat{h}}^{2}}}, \\
\Gamma\left(\hat{h} \rightarrow \hat{\chi}_{0}\right)=\Gamma(\hat{h} \rightarrow \hat{b} \hat{b})\left\langle N_{[\hat{b} \hat{b}]}\right\rangle \hat{\kappa}_{0} \sqrt{1-\frac{4 m_{\hat{\chi}}^{2}}{m_{\hat{h}}^{2}}},
\end{gathered}
$$

where $\left\langle N_{\hat{G}}\right\rangle$ and $\left\langle N_{[\hat{b} \hat{b}]}\right\rangle$ are the hadronic multiplicities of glueball and bottomonium states at the heavy Higgs mass $m_{\hat{h}}$, respectively. Whereas, $\hat{\kappa}_{0}$ is the fraction of glueball or bottomonium states in the $0^{++}$state. Twin QCD hadronic average multiplicity $\left\langle N_{\text {had }}\right\rangle$ is defined as [61],

$$
\left\langle N_{\text {had }}(s)\right\rangle=N_{0} \exp \left[\frac{1}{\hat{b}_{0}} \sqrt{\frac{6}{\pi \hat{\alpha}_{\mathrm{QCD}}(s)}}+\left(\frac{1}{4}+\frac{5 n_{\hat{f}}}{54 \pi \hat{b}_{0}}\right) \ln \hat{\alpha}_{\mathrm{QCD}}(s)\right],
$$

where $\hat{b}_{0}=\left(33-2 n_{\hat{f}}\right) /(12 \pi)$ is the twin QCD beta function, $\hat{\alpha}_{\mathrm{QCD}}(s)$ is the twin QCD coupling evaluated at the center-of-mass energy $\sqrt{s}$, and $n_{\hat{f}}$ are the number of twin quark flavors below the confinement scale $\hat{\Lambda}_{\mathrm{QCD}}$. The normalization constant $N_{0}$ is a free parameter which we fix by setting the number of twin hadrons at a fixed low energy. For example, for a glueball of mass $m_{\hat{0}^{+}}=50 \mathrm{GeV}$, a scalar at mass $125 \mathrm{GeV}$ can produce roughly two glueballs, so we fix $N_{0}$ by the condition $\left\langle N_{\hat{G}}\right\rangle_{125}=2$. The above hadron multiplicity for the dark QCD has been tested by [62] with showering/hadronization in PYTHIA [63] and a very good agreement has been found.

In figure 10 (left-panel) we plot the average multiplicity of twin glueballs as a function of the twin Higgs mass for different choices of the lightest glueball mass $m_{\hat{0}^{+}}$. Note that we 

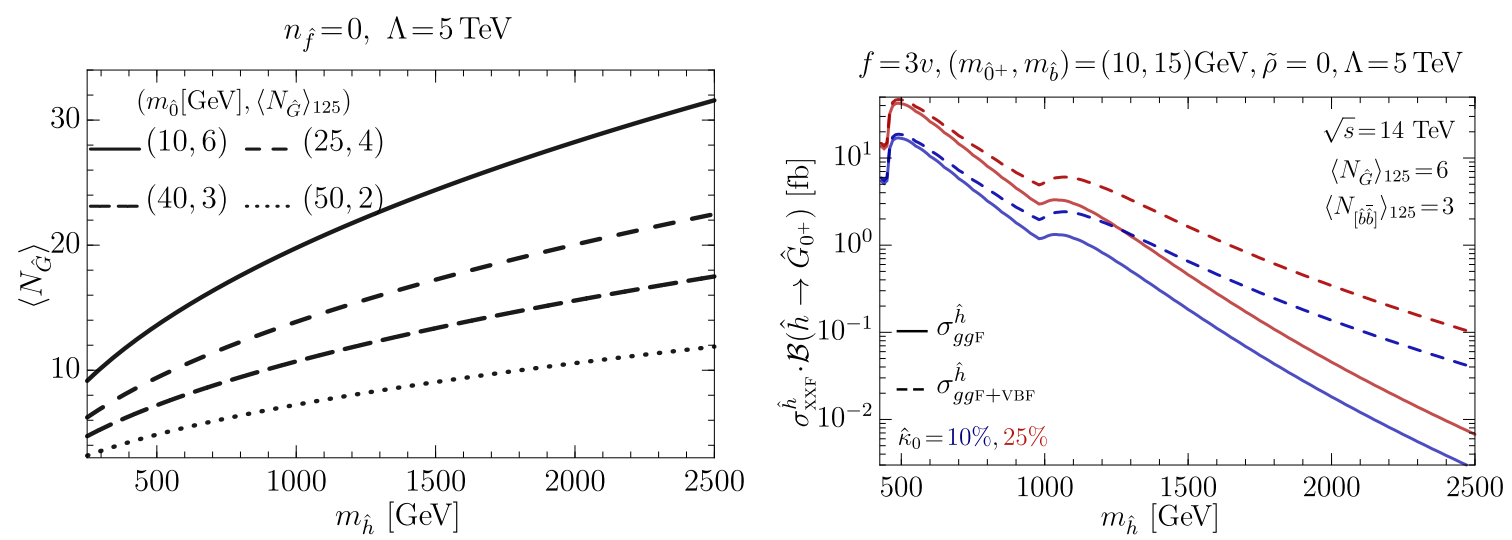

Figure 10. The left-graph shows average glueball multiplicity $\left\langle N_{\hat{G}}\right\rangle$ as a function of the heavy Higgs mass for different choices of the lightest glueball mass and the corresponding normalized number of glueball states at the SM Higgs mass. In the right-panel we plot cross-section times branching fraction of the heavy twin Higgs decaying to the lightest glueballs $\hat{G}_{0^{+}}$with mass $m_{\hat{0}^{+}}=10 \mathrm{GeV}$ as a function of the twin Higgs mass. The twin Higgs at the $14 \mathrm{TeV}$ LHC is produced via ggF (solid) and $g g \mathrm{~F}+\mathrm{VBF}$ (dashed). We take $\hat{\kappa}_{0}=10 \%$ (blue) and $\hat{\kappa}_{0}=25 \%$ (red) for the lightest glueball multiplicity.

normalize the glueball multiplicity w.r.t. to the SM Higgs, i.e. we take $\left\langle N_{\hat{G}}\right\rangle_{125}=(6,4,3,2)$ corresponding to $m_{\hat{0}^{+}}=(10,25,40,50) \mathrm{GeV}$, respectively, which are rather conservative numbers. Moreover, when the twin bottomonium states are heavier than the lightest glueball, then through the relaxation/annihilation these bottomonium states decay to twin gluons which then hadronize to form the glueballs. In the following we assume if $m_{[\hat{b} \hat{b}]}>$ $2 m_{\hat{0}^{+}}$then all the bottomonium states decay to the glueballs, i.e. $[\hat{b} \hat{b}] \rightarrow$ glueballs. The right-panel of figure 10 show the production cross-section of the lightest glueball state with mass $m_{\hat{0}^{+}}=10 \mathrm{GeV}$ via the heavy twin Higgs. The heavy Higgs production is considered by the ggF (solid curve) and ggF+VBF (dashed curve) at the LHC with center-of-mass energy $14 \mathrm{TeV}$. As mentioned above, around a dozen (depending on their masses) metastable glueball states are formed and the probability to produce the lightest glueball $\hat{G}_{0^{+}}$is parameterized by $\hat{\kappa}_{0}$, we take a conservative choice of $\hat{\kappa}_{0}=10 \%$ (blue curves) and $\hat{\kappa}_{0}=25 \%$ (red curves). In figure 10 (right-panel) we consider $m_{\hat{b}}=15 \mathrm{GeV}\left(n_{\hat{f}}=0\right)$, such that only stable twin hadrons are the glueballs, whereas, other parameters are $f / v=3$ and $\tilde{\rho}=0$. The bumps in the curves of glueball cross-section indicate thresholds for the twin $\hat{Z}$ and top quark $\hat{t}$, as the decays of twin $\hat{Z}$ boson and $\hat{t}$ quark to twin bottom $\hat{b}$ quarks lead to hadronize and decay to the glueball states. Note that there could be non-perturbative effects in the production of the twin glueballs as large as $10 \%$ as argued in [5], however, we assume they are within the uncertainty of our approximation for the average hadron multiplicity and the $\hat{\kappa}_{0}$ parameter. Nevertheless, the above parameterization provides a reasonable estimate for the production of fraternal twin hadrons.

The lightest twin glueball $\hat{G}_{0^{+}}$mixes with the TH scalar sector, which leads to its decay to the SM light quarks and leptons, see figure 11 (left). In the following, we calculate decay width of the twin glueball $\hat{G}_{0^{+}}$to the SM light fermions. The first step is to calculate the 

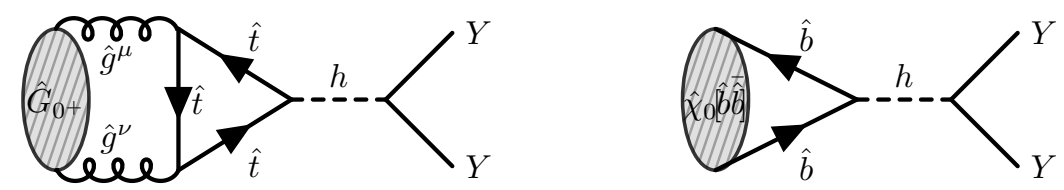

Figure 11. Schematic diagrams of twin glueball $\hat{G}_{0^{+}}$and twin bottomonium $\hat{\chi}_{0}$ decays to the SM states $Y$ (mostly bottom quarks) via an off-shell SM Higgs $h$.

twin gluon coupling to the SM Higgs,${ }^{6}$ which is given by,

$$
\mathcal{L}_{h h \hat{g} \hat{g}}^{\text {int }}=c_{\hat{g} \hat{g}} \hat{g}_{h} \operatorname{Tr}\left[G_{\mu \nu}^{a} G^{a, \mu \nu}\right] h, \quad \text { where } \quad c_{\hat{g} \hat{g}} \equiv-\frac{\hat{\alpha}_{\mathrm{QCD}}}{4 \pi \hat{v}} \sum_{i} \hat{F}_{1 / 2}\left(\tau_{i}\right),
$$

where $i=\hat{t}, \hat{b}$ (dominant contribution is by twin top loop) and in the limit $\tau_{\hat{t}}=4 m_{\hat{t}}^{2} / m_{h}^{2} \gg$ 1 the triangle loop function $\hat{F}_{1 / 2}\left(\tau_{\hat{t}}\right) \rightarrow-4 / 3$, whereas $\hat{g}_{h}=-\sin \alpha$ (see also figure 16). Hence in the low-energy effective theory, twin gluon coupling to the SM Higgs is

$$
\mathcal{L}_{h \hat{g} \hat{g}}^{\operatorname{int}}=-\frac{\hat{\alpha}_{\mathrm{QCD}}}{3 \pi \hat{v}} \sin \alpha \operatorname{Tr}\left[G_{\mu \nu}^{a} G^{a, \mu \nu}\right] h .
$$

Now following [11] we can write decay rate of the glueball to the SM light states $Y$ as,

$$
\Gamma\left(\hat{G}_{0^{+}} \rightarrow Y Y\right)=\left.\left(\frac{\hat{\alpha}_{\mathrm{QCD}} \sin 2 \alpha \mathbf{F}_{\hat{0}^{+}}}{6 \pi \hat{v}\left(m_{h}^{2}-m_{\hat{0}^{+}}^{2}\right)}\right)^{2} \Gamma^{\mathrm{SM}}(h \rightarrow Y Y)\right|_{m_{h}=m_{\hat{0}^{+}}},
$$

where $\mathbf{F}_{\hat{0}^{+}} \equiv\left\langle 0\left|\operatorname{Tr}\left[\hat{G}_{\mu \nu}^{a} \hat{G}^{a, \mu \nu}\right]\right| \hat{0}^{++}\right\rangle$is the decay constant of the lightest glueball $\hat{G}_{0^{+}}$and its value by lattice is $4 \pi \hat{\alpha}_{\mathrm{QCD}} \mathbf{F}_{\hat{0}^{+}}=3.06 m_{\hat{0}^{+}}^{3}$ [64]. Above, $\Gamma^{\mathrm{SM}}(h \rightarrow Y Y)$ is the SM Higgs decay rate to the SM light states $Y$ evaluated at the twin glueball mass $m_{\hat{0}^{+}}$.

Assuming the twin leptons $(\hat{\ell}=\hat{\tau}, \hat{\nu})$ are heavy enough such that the invisible twin weak decays of $\hat{\chi}_{0} \rightarrow \hat{\Upsilon} \hat{\ell} \hat{\ell}$ are kinematically forbidden. Then, as mentioned above, for $m_{\hat{\chi}}>2 m_{\hat{0}^{+}}$, bottomonium state $\hat{\chi}_{0}$ decays to the glueballs, otherwise for $m_{\hat{\chi}}<2 m_{\hat{0}^{+}}$, $\hat{\chi}_{0}$ decays to the visible sector fermions. The decays of $\hat{\chi}_{0}$ state to the SM sector would meditate through the SM Higgs, as shown in figure 11 (right), and the interaction is given by,

$$
\mathcal{L}_{h \hat{b} \hat{b}}^{\text {int }}=-\frac{y_{\hat{b}}}{\sqrt{2}} \hat{g}_{h} h \hat{b} \overline{\hat{b}}=\frac{m_{\hat{b}}}{\hat{v}} \sin \alpha h \hat{b} \hat{\hat{b}} .
$$

An estimate of the bottomonium state $\hat{\chi}_{0}$ decay to the SM light fermions via an off-shell Higgs using non-relativistic quantum mechanism is [5],

$$
\Gamma\left(\hat{\chi}_{0} \rightarrow Y Y\right)=\left.\frac{27}{4 \pi}\left|R^{\prime}(0)\right|^{2} \frac{\sin ^{2}(2 \alpha) m_{\hat{\chi}}^{2}}{32 \hat{v}^{2}\left(m_{h}^{2}-m_{\hat{\chi}}^{2}\right)^{2}} \Gamma^{\mathrm{SM}}(h \rightarrow Y Y)\right|_{m_{h}=m_{\hat{\chi}}},
$$

where $R^{\prime}(0)$ is derivative of the radial wave-function of p-wave state $\hat{\chi}_{0}$ w.r.t. its radius. We use the estimate of $\left|R^{\prime}(0)\right|^{2}$ given by [5], which employs linear confining potential, but

\footnotetext{
${ }^{6}$ Note that the twin $0^{++}$hadrons also decay to the SM light fermions via an off-shell heavy twin Higgs $\hat{h}$, however, these processes can be neglected as they are suppressed by $\left(m_{h} / m_{\hat{h}}\right)^{4}$ in comparison to the SM Higgs mediated decays.
} 
neglecting the relativistic corrections which could be potentially important. The estimate of $\left|R^{\prime}(0)\right|^{2}$ for $\hat{\chi}_{0}$ state is [5],

$$
\left|R^{\prime}(0)\right|^{2} \approx 0.002 \frac{m_{\hat{\chi}}^{5 / 3} m_{\hat{0}^{+}}^{10 / 3}}{m_{h}} .
$$

In the following subsection we calculate the production cross-section for the twin hadrons via heavy twin Higgs in $m_{\hat{h}}-f / v$ plane. The decay length of the twin $0^{++}$ hadron is,

$$
c \tau_{0^{++}}=\frac{\hbar c}{\Gamma_{0^{++}}} \text {. }
$$

Depending on kinematics of the produced twin hadrons $\left(0^{++}\right)$their decay-length $c \tau_{0^{++}}$ can be from very short $\lesssim 10^{-3}[\mathrm{~m}]$ to very long $\gtrsim 10^{2}[\mathrm{~m}]$. For very short decay-length the daughter decays to the SM light fermions would be prompt, so the signature at the colliders could be high multiplicity jets, see e.g. [65, 66]. On the contrary, for very long decay-length the twin-hadrons would escape the detectors and signatures would be missing energy. However, the most promising signals would appear for the case when the twin hadrons decay-length is of the size of detectors, i.e. $10^{-3} \mathrm{~m} \lesssim c \tau_{0^{++}} \lesssim 10^{2} \mathrm{~m}$, such that the signatures would appear as displaced vertices at the colliders. There are ongoing searches at the LHC for long-lived particles at the ATLAS [67-69], the CMS [70, 71] and the LHCb [72] experiments.

\subsection{Fraternal Twin hadron phenomenology}

In this subsection, we calculate the production cross-section of twin glueball $\hat{G}_{0^{+}}$and twin bottomonium $\hat{\chi}_{0}$ states via heavy twin Higgs at the $14 \mathrm{TeV}$ LHC. ${ }^{7}$ For this purpose, we choose several benchmark cases where either the lightest twin hadron is a glueball or a bottomonium state.

In figure 12 we plot the contours of production cross-section [fb] (solid blue) and proper decay-length $[\mathrm{m}]$ (dashed green) of glueballs $\hat{G}_{0^{+}}$with mass $m_{\hat{0}^{+}}=10 \mathrm{GeV}$ in $m_{\hat{h}}-\mathrm{f} / \mathrm{v}$ plane, and the twin bottom quark mass $m_{\hat{b}}=15 \mathrm{GeV}$. The left and right panels of figure 12 are for $\tilde{\rho}=0 \mathrm{GeV}$ and $\tilde{\rho}=0.5 \mathrm{GeV}$, respectively. For the chosen masses of twin hadrons we normalize average hadron multiplicity at the SM Higgs mass as $\left\langle N_{\hat{G}}\right\rangle_{125}=6$ and $\left\langle N_{[\hat{b} \hat{b}]}\right\rangle_{125}=3$ for the twin glueball and bottomonium states, respectively. Note that for $m_{\hat{b}}=15 \mathrm{GeV}$ the twin bottomonium states with mass $m_{[\hat{b} \hat{b}]} \simeq 30 \mathrm{GeV}$ are unstable and we assume they decay $100 \%$ to the twin glueballs. Moreover, we set $\hat{\kappa}_{0}=0.25$, the probability to produce the lightest glueball state $\hat{G}_{0^{+}}$. The shaded regions correspond to the unphysical parameters (gray), exclusion by the SM Higgs signal-strength measurements (red), and exclusion by the SM invisible branching fraction (hatched light-gray). Notice that heavy twin Higgs cross-section to twin glueballs $\hat{G}_{0^{+}}$is comparable to its cross-section to the SM Higgs/vector bosons. The decay length of twin glueball $\hat{G}_{0^{+}}$with mass $m_{\hat{0}^{+}}=10 \mathrm{GeV}$ is rather large $\gtrsim 10 \mathrm{~m}$ in most of the FTH parameter space, so it decays outside the detector and hence would appear as a missing energy signal.

\footnotetext{
${ }^{7}$ In this work, we do not discuss the production of twin hadrons via the SM Higgs in the FTH model, however, we refer to refs. [39-42].
} 

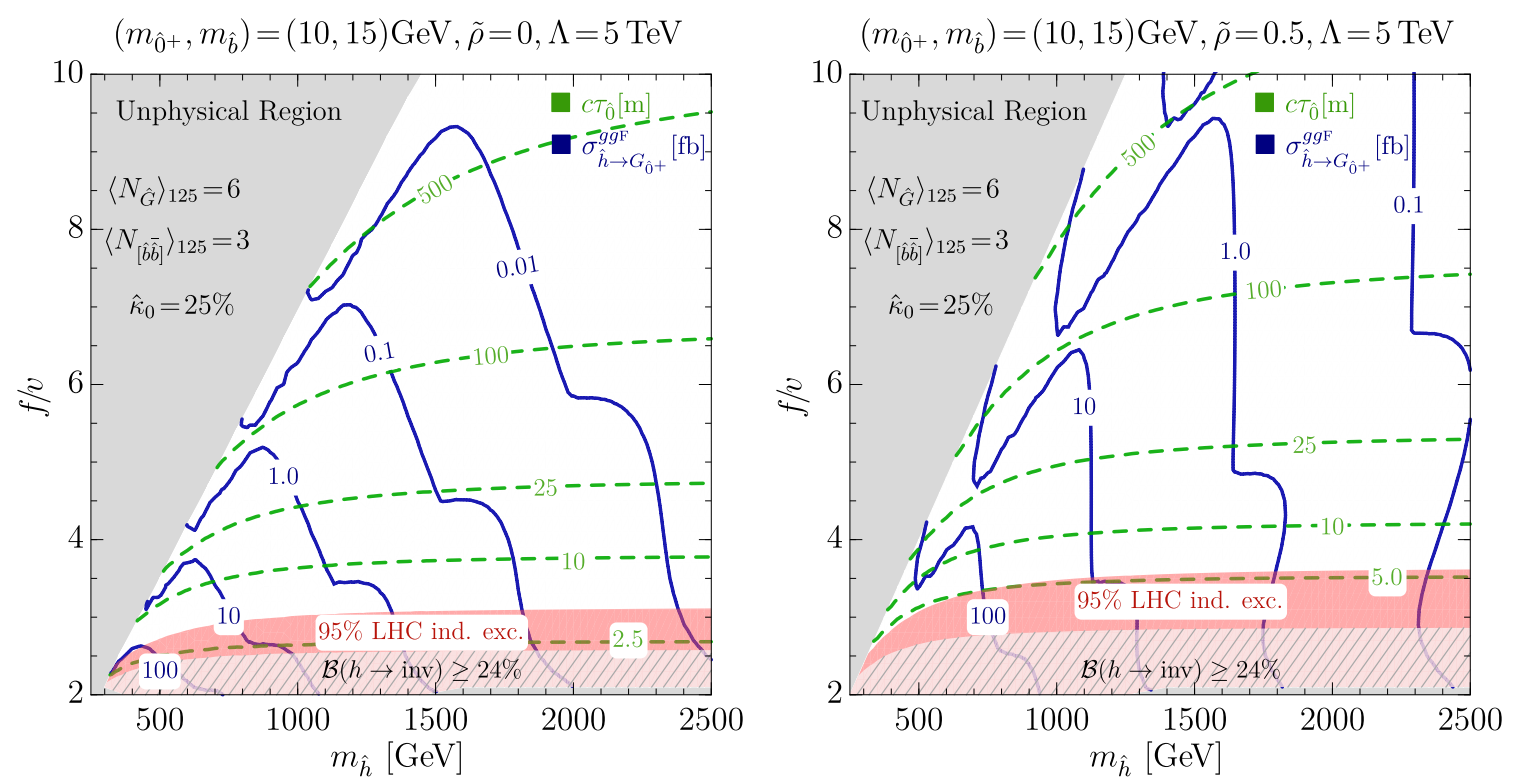

Figure 12. These plots show parameter space in $m_{\hat{h}}-f / v$ plane for the FTH model. The contours of heavy twin Higgs cross-section to twin glueball $\hat{G}_{0^{+}}$with mass $m_{\hat{0}^{+}}=10 \mathrm{GeV}$ (solid blue) and the glueball decay-length $c \tau_{\hat{0}}$ (dashed green) are shown for $\tilde{\rho}=0$ (left) and $\tilde{\rho}=0.5$ (right).

For $0^{++}$twin glueballs with masses $m_{\hat{0}^{+}}=25 \mathrm{GeV}$ and $50 \mathrm{GeV}$ we show the contours of their production cross-sections (solid blue) and proper decay-lengths (dashed green) in $m_{\hat{h}}-f / v$ plane in the left- and right-panel of figure 13, respectively. Whereas, in figure 13 , we fix the twin bottom quark mass $m_{\hat{b}}=75 \mathrm{GeV}$, such that they annihilate to the twin glueballs. For the above masses of twin hadrons we normalized the average hadron multiplicity at $200 \mathrm{GeV}$ as $\left\langle N_{\hat{G}}\right\rangle_{200}=6$ for $m_{\hat{0}^{+}}=25 \mathrm{GeV}$ and $\left\langle N_{\hat{G}}\right\rangle_{200}=3$ for $m_{\hat{0}^{+}}=50 \mathrm{GeV}$, and $\left\langle N_{[\hat{b} \hat{b}]}\right\rangle_{200}=1$. Notice for glueball mass $m_{\hat{0}^{+}}=25 \mathrm{GeV}$ (left-panel of 13), its decay-length is in the range $10^{-2} \mathrm{~m} \lesssim c \tau_{0^{++}} \lesssim 1 \mathrm{~m}$, which is an ideal range for the observation of displaced vertices at the LHC [67-72]. For glueball mass $m_{\hat{0}^{+}}=50 \mathrm{GeV}$ (right-panel of 13), the decay-length is $10^{-5} \mathrm{~m} \lesssim c \tau_{0^{++}} \lesssim 10^{-3} \mathrm{~m}$, which would give prompt decays, however, as we have neglected any effects due to boosted production of the twin hadrons via heavy twin Higgs decays, which could potentially increase the decay-length.

In figure 14, we consider the case when the twin glueballs are heavier than the light bottomuium states such that they decay to two bottomonium states, i.e. glueballs $\rightarrow[\hat{b} \hat{b}][\hat{b} \hat{b}]$. In particular, we consider two cases with $\left(m_{\hat{0}^{+}}, m_{\hat{\chi}}\right)=(50,25) \mathrm{GeV}$ (left-panel) and $\left(m_{\hat{0}^{+}}, m_{\hat{\chi}}\right)=(100,40) \mathrm{GeV}$ (right-panel), where contours of the cross-section and the decaylength of twin bottomonium state $\hat{\chi}_{0}$ are shown. We normalize the average multiplicity at the SM Higgs mass as $\left(\left\langle N_{\hat{G}}\right\rangle_{125},\left\langle N_{[\hat{b} \hat{b}]}\right\rangle_{125}\right)=(2,4)$ and $(1,2)$ for the twin hadron masses $\left(m_{\hat{0}^{+}}, m_{\hat{\chi}}\right)=(50,25) \mathrm{GeV}$ and $(100,40) \mathrm{GeV}$, respectively. Moreover, for the probability to produce $0^{++}$bottomonium state $\hat{\chi}$ we take $\hat{\kappa}_{0}=0.25$. Note that the twin bottomonium decay-length is in a range to give displaced vertices at the LHC.

Current LHC searches [67-72] for long-lived particles are not sensitive for the crosssections obtained here for the twin hadron production via heavy twin Higgs so we do not get 

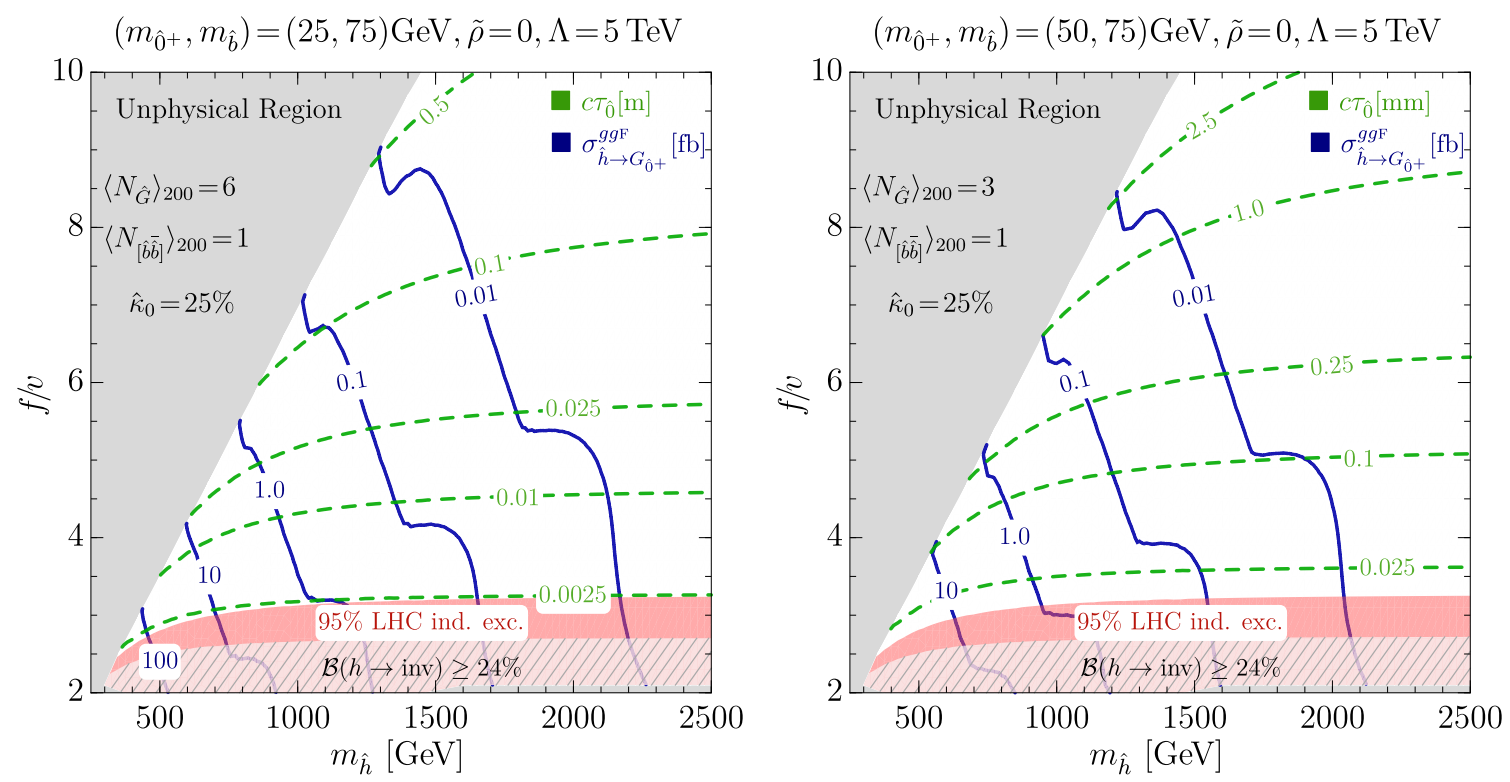

Figure 13. These graphs show the contours of production cross-sections (solid blue) and proper decay-lengths (dashed green) of twin glueball $\hat{G}_{0^{+}}$with mass $m_{\hat{0}^{+}}=25 \mathrm{GeV}$ and $50 \mathrm{GeV}$ in the leftand right-panel, respectively, with $m_{\hat{b}}=75 \mathrm{GeV}$ and $\tilde{\rho}=0$.
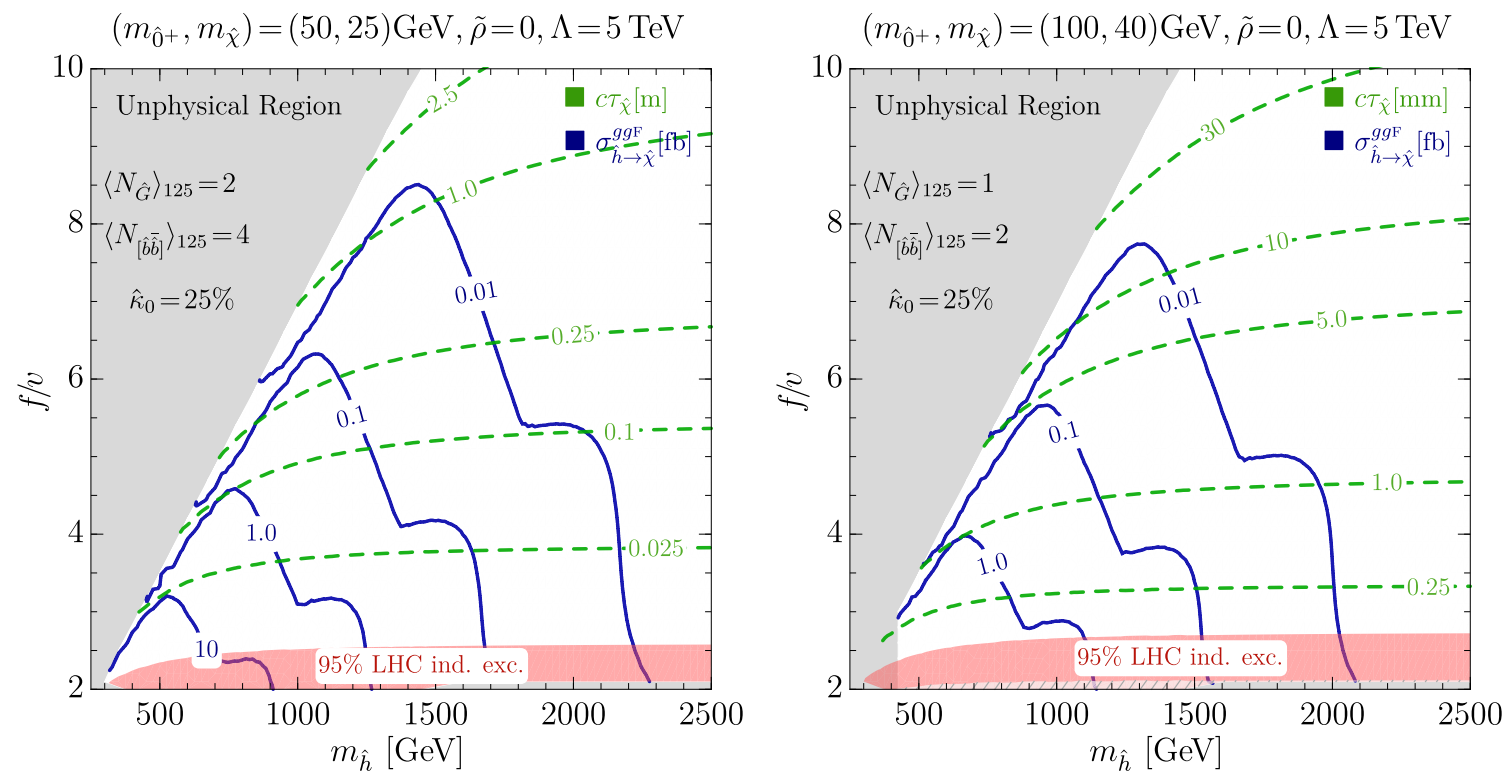

Figure 14. These graphs show the contours of cross-sections (solid blue) and decay-lengths (dashed green) of twin bottomonium state $\hat{\chi}_{0}$ with mass $m_{\hat{\chi}}=25 \mathrm{GeV}$ and $40 \mathrm{GeV}$ in the left- and rightpanel, respectively. Moreover, we consider $m_{\hat{0}^{+}}=50 \mathrm{GeV}$ (left) and $100 \mathrm{GeV}$ (right) for $\tilde{\rho}=0$. 
any constraints, however in future some of the regions of the FTH parameter space can be probed by these searches. The studies of twin hadron production via the SM Higgs for the FTH model seems to offer more feasibility for the observation of twin hadrons at the LHC, see [39-42]. Projected reach for the HL-LHC is to explore the TH parameter space up to $f / v \lesssim 6$ in the twin glueball mass window $m_{\hat{0}^{+}} \approx[10,60],[39]$. However, the twin hadron production through the SM Higgs is limited only for the low mass $\left(\lesssim m_{h} / 2\right)$, whereas, their production via heavy Higgs decay offers chances to probe heavier twin hadrons as well.

\subsection{Heavy Twin Higgs phenomenology}

In this subsection, we briefly discuss the direct and indirect phenomenological prospects of the heavy twin Higgs in the weak sector. As we have learned from figure 8, the dominant decay modes of the twin Higgs in the FTH model are the SM and twin massive gauge bosons and the SM Higgs. Roughly speaking, phenomenology of heavy twin Higgs in the FTH model is very similar to that of the MTH case. The only difference, which can be crucial for indirect constraints, is the flexibility of twin light fermion masses which may enhance the SM Higgs invisible decay width.

We consider one benchmark scenario for the choice of twin glueball mass $m_{\hat{0}^{+}}=25 \mathrm{GeV}$ and twin bottom mass $m_{\hat{b}}=75$. Moreover, we assume that twin lepton mass (Yukawa) scale as $m_{\hat{\tau}}=\frac{m_{\hat{b}}}{m_{b}} m_{\tau}$. In the left-panel of figure 15 we plot contours of the twin Higgs crosssection (solid blue) to a pair of SM $Z$ bosons and the SM Higgs signal-strength $\mu_{h \rightarrow Z Z}^{g g \mathrm{~F}}$ (dashed red) for $\tilde{\rho}=0$ in $m_{\hat{h}}-f / v$ plane. The right-panel of figure 15 shows the concours of the ratio of heavy twin Higgs width over its mass $\Gamma_{\hat{h}} / m_{\hat{h}}$ (solid orange), the SM Higgs trilinear coupling over its SM value $g_{h h h} / g_{h h h}^{\mathrm{SM}}$ (shaded purple), and the SM Higgs invisible (twin sector) branching ratio $\mathcal{B}(h \rightarrow$ inv.) (dotted gray). The shaded regions represent: unphysical parameters (gray), exclusion by the SM Higgs signal-strength measurements (red), exclusion by the SM invisible branching fraction (hatched light-gray), exclusion by the EWPT at $95 \%$ C.L. (below the gray line, hatched area), exclusion by the direct heavy scalar searches at the LHC (purple), and the HL-LHC projected reach for direct searches (light blue). Note that for our choice of twin bottom mass $m_{\hat{b}}=75$, we have $m_{\hat{\tau}} \simeq 30 \mathrm{GeV}$, which implies that the SM Higgs decays to $\hat{b} \hat{b}$ are forbidden but to $\hat{\tau} \overline{\hat{\tau}}$ are allowed. The invisible decays of the SM Higgs are only in twin gluonic and twin leptonic channels.

\section{Conclusions}

In this work, we have explored phenomenological implications of the radial mode of Twin Higgs models, when the TH symmetry breaking is linearly realized. We consider an SU(4) global symmetry for TH models which is spontaneously broken to $\mathrm{SU}(3)$ and gives $\mathbf{7}$ Goldstone bosons. Out of these $\mathbf{7}$ Goldstone bosons, $\mathbf{6}$ are "eaten" by the SM and twin weak gauge bosons, and hence leaving only one Goldstone boson which plays the role of the SM Higgs boson. We consider an EFT approach and employ the most general symmetry breaking structure for the TH scalar potential including explicit soft and hard breaking $\mathbb{Z}_{2}$ discrete and SU(4) global symmetries. These explicit breaking terms are the source of generating the SM Higgs mass. These terms are model dependent quantities and are 

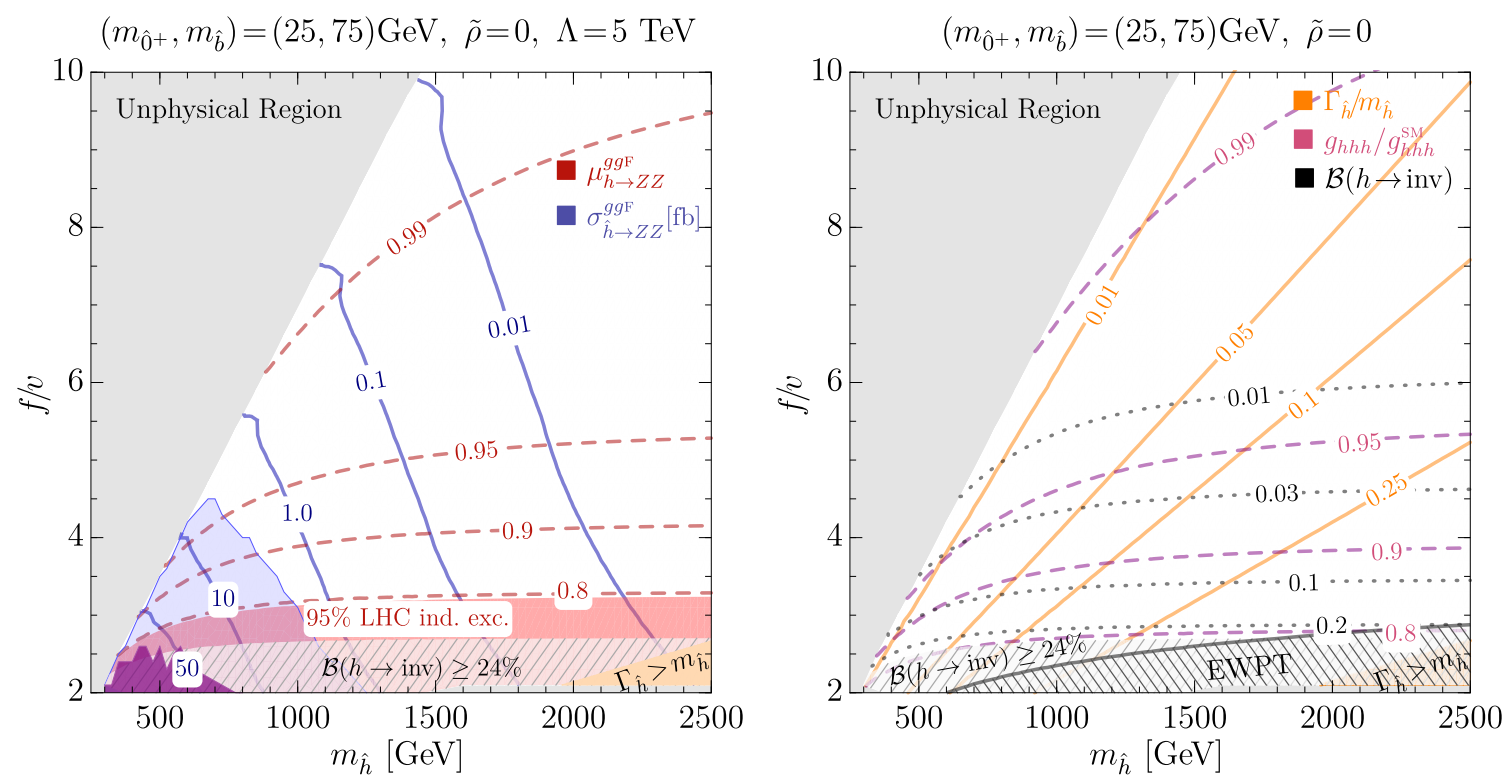

Figure 15. The left-panel shows the contours of the cross-section of the heavy Higgs to the SM vector bosons (solid blue) and the SM Higgs signal-strength (dashed red) in $m_{\hat{h}}-f / v$ plane of the FTH model with $\tilde{\rho}=0$. The right-plot show the contours of the ratio of heavy twin Higgs width over mass, $\Gamma_{\hat{h}} / m_{\hat{h}}$ (solid orange), the ratio of the SM-like Higgs trilinear coupling over its corresponding SM values, $g_{h h h} / g_{h h h}^{\mathrm{SM}}$ (dashed purple), and the SM Higgs invisible (twin) branching fraction $\mathcal{B}\left(h \rightarrow\right.$ inv.) (dotted). For these plots we take the twin glueball mass $m_{\hat{0}^{+}}=25 \mathrm{GeV}$ and the twin bottom quark mass $m_{\hat{b}}=75 \mathrm{GeV}$, whereas the details of shaded regions are given in the text.

calculable in a given perturbative UV complete TH model. The mixing of the SM-like Higgs and the twin Higgs (radial mode) provide a portal to the SM and twin sectors, which could potentially lead to the discovery of TH mechanism.

We have considered two Twin Higgs scenarios; the MTH, which has an exact mirror of the SM field content in the twin sector, and the FTH, which has the minimal field content needed to solve the little hierarchy problem. The symmetry breaking pattern of the TH mechanism is such that the SM Higgs and weak gauge bosons, and twin weak gauge bosons are the Goldstone bosons of same broken symmetry, therefore their interactions with the radial mode (heavy twin Higgs) are fixed. As a result, the heavy twin Higgs branching fractions to the SM Higgs and weak gauge bosons $W^{ \pm}, Z$ are equal. Furthermore, the twin Higgs branching fractions to the SM sector as compared to the twin sector are approximately $\sim 4 / 3$. However, as shown in sections 3 and 4 , this universal behavior changes considerably when explicit hard breaking is turned on. Employing the current experimental direct and indirect searches at the LHC for a heavy scalar we explore the parameter space of the TH models. Current LHC direct searches for a heavy scalar to a pair of weak gauge boson constraint a small region of the MTH parameter space, in particular excluding the twin Higgs $\lesssim 500 \mathrm{GeV}$ mass. Whereas, the searches in the other decay channels especially to a pair of SM Higgs are not constraining with the available data. However, it is shown that the HL-LHC reach, at $14 \mathrm{TeV}$ with $3000 \mathrm{fb}^{-1}$, can discover/exclude the heavy twin 
Higgs up to $1 \mathrm{TeV}$ mass. On the other hand, indirect searches at the LHC by the SM Higgs signal-strength measurements are quite constraining. In particular, the current LHC data excludes $f / v \lesssim 3$ without explicit hard breaking contributions. In the presence of explicit hard breaking contributions, the $f / v$ ratio could be less or more constrained depending on the sign of these terms. Moreover, the HL-LHC reach for the SM Higgs signal-strength measurements could potentially exclude the MTH parameter space up to $f / v \lesssim 6$.

In section 4, we have explored phenomenology of the twin Higgs in the FTH model. In particular, we have discussed the consequences of twin QCD confinement. In the absence of light quark flavors in the FTH model, twin QCD confinement/hadronization leads to the formation of twin glueball/bottomonium states, which have interesting phenomenological signatures. We have discussed the production of these twin hadrons via heavy twin Higgs decays at the LHC. More importantly, the $0^{++}$glueball $\hat{G}_{0^{+}}$and bottomonium $\hat{\chi}_{0}$ states have the correct quantum number to mix with the scalar sector of the model, as a result, decays of these twin hadrons back to the SM light fermions are possible. The lessons we have learned from the twin hadron phenomenology are: (i) The heavy Higgs cross-section to the $0^{++}$twin hadrons at the LHC is comparable to that of the SM vector bosons, and (ii) Depending on the masses of $0^{++}$twin hadrons, their decays can be prompt, displaced or even can escape the detectors. These signatures offer a unique experimental opportunity to search for multi-jet prompt or displaced objects and/or missing energy signals at the colliders. Hence, any signals of such events at the LHC could lead to the discovery of twin Higgs framework.

\section{Acknowledgments}

The author thanks Zackaria Chacko and Saereh Najjari for discussions and comments on the draft, and also informing about their related work [73]. He also thanks Barry Dillon and Bohdan Grzadkowski for useful comments. He is grateful to the Mainz Institute for Theoretical Physics (MITP) and the Laboratoire de Physique Théorique (LPT) Orsay, for their hospitality and support while this work was in progress. This work was partially supported by the National Science Centre (Poland) research projects, decision DEC-2014/13/B/ST2/03969, and DEC-2014/15/B/ST2/00108.

\section{A Twin Higgs Feynman rules and partial decay widths}

In this appendix, we collect partial decay widths of the heavy twin Higgs employed in the main text. The most relevant Feynman rules for the scalar sector of the TH models are collected in figure 16, where the triangle loop functions are given below.

Heavy Higgs decays into fermions. The two body decays of heavy twin Higgs into the SM and twin sector fermions are given as:

$$
\Gamma^{\hat{h}}(f f)=\frac{G_{\mathrm{F}} g_{\hat{h}}^{2}}{4 \sqrt{2} \pi} m_{\hat{h}} m_{f}^{2}\left(1-\frac{4 m_{f}^{2}}{m_{\hat{h}}^{2}}\right)^{3 / 2}, \quad \Gamma^{\hat{h}}(\hat{f} \hat{f})=\frac{\hat{G}_{\mathrm{F}} \hat{g}_{\hat{h}}^{2}}{4 \sqrt{2} \pi} m_{\hat{h}} m_{\hat{f}}^{2}\left(1-\frac{4 m_{\hat{f}}^{2}}{m_{\hat{h}}^{2}}\right)^{3 / 2}
$$




\begin{tabular}{|c|c|}
\hline SM SECTOR & TWIN SECTOR \\
\hline$-i \frac{m_{f}}{v} g_{h, \hat{h}}, \quad$ f: SM fermions & $-i \frac{m_{\hat{f}}}{\hat{v}} \hat{g}_{h, \hat{h}},{ }_{\hat{f}}:$ Twin fermions \\
\hline$\underbrace{i \frac{2 m_{V}^{2}}{v} g_{h, \hat{h}} \eta^{\mu \nu},}_{V^{\nu}}, \quad V: W, Z$ & $\begin{array}{l}\boldsymbol{\tau}_{\hat{V}^{\nu}} \hat{V}^{\mu} \\
i \frac{2 m_{\hat{v}}^{2}}{\hat{v}} \hat{g}_{h, \hat{h}} \eta^{\mu \nu} \\
\hat{V}: \hat{W}, \hat{Z}\end{array}$ \\
\hline 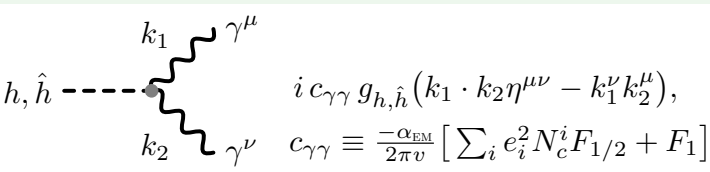 & 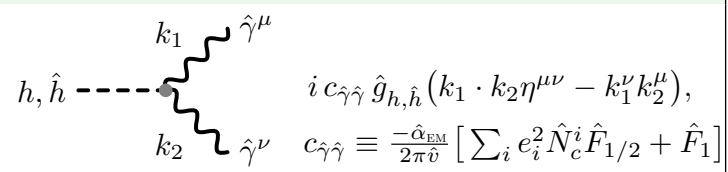 \\
\hline 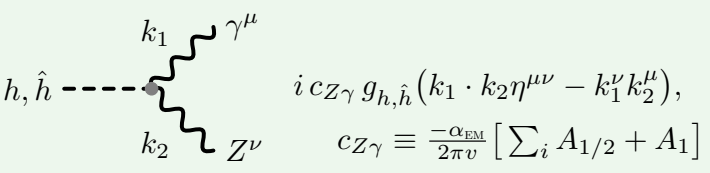 & 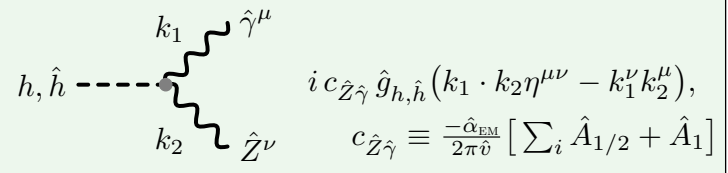 \\
\hline 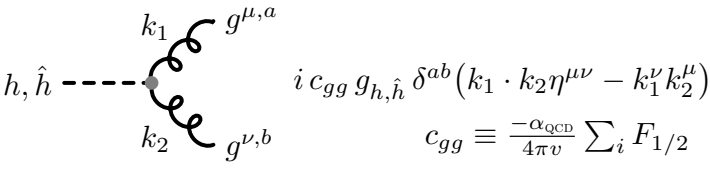 & 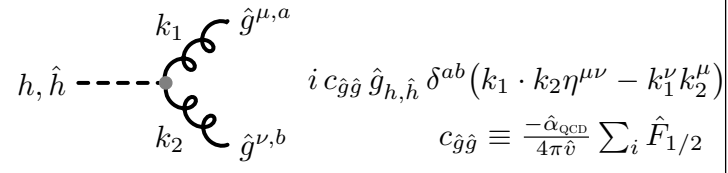 \\
\hline $\begin{aligned}-i g_{\hat{h} h h} & \equiv-i 2\left[\hat{v} \cos \alpha\left(\lambda+3 \kappa \sin ^{2} \alpha\right)\right. \\
& \left.+v \sin \alpha\left(\lambda+3(\kappa+\rho) \cos ^{2} \alpha\right)\right]\end{aligned}$ & $\begin{aligned} \text { where } & g_{h}=\hat{g}_{\hat{h}} \equiv \cos \alpha, & & g_{\hat{h}}=-\hat{g}_{h} \equiv \sin \alpha \\
\hat{v} & =f \sqrt{1-\xi^{2}}, & & \xi \equiv v / f\end{aligned}$ \\
\hline
\end{tabular}

Figure 16. Feynman rules for the interactions of SM and twin sector particles with the SM Higgs $h$ and heavy twin Higgs $\hat{h}$. The SM triangle loop functions $F_{1 / 2}, F_{1}, A_{F}$ and $A_{W}$ are given below and the corresponding twin triangle loop functions $\hat{F}_{1 / 2}, \hat{F}_{1}, \hat{A}_{F}$ and $\hat{A}_{W}$ has similar form as the SM ones but with twin particles in the loops.

where $g_{\hat{h}}=\sin \alpha, \hat{g}_{\hat{h}}=\cos \alpha$, and twin Fermi constant is $\hat{G}_{\mathrm{F}}=\left(v^{2} / \hat{v}^{2}\right) G_{\mathrm{F}}$. Moreover, in our numerical analysis we have allowed off-shell decays of the heavy fermions (the SM and the twin top quarks).

Heavy Higgs decays into gauge bosons. The heavy twin Higgs partial decay width to on-shell SM massive gauge bosons is,

$$
\Gamma^{\hat{h}}\left(V_{1} V_{2}\right)=\frac{G_{\mathrm{F}} m_{\hat{h}}^{3} g_{\hat{h}}^{2}}{8 \sqrt{2} \pi \mathcal{S}_{V}} \lambda\left(x_{1}, x_{2}\right)\left[\lambda^{2}\left(x_{1}, x_{1}\right)+12 x_{1} x_{2}\right]
$$

where, $\lambda\left(x_{1}, x_{2}\right) \equiv \sqrt{\left(1-x_{1}-x_{2}\right)^{2}-4 x_{1} x_{2}}$ with $x_{1,2}=m_{V_{1,2}}^{2} / m_{\hat{h}}^{2}$ and $\mathcal{S}_{V}$ is a symmetry factor, for identical gauge bosons it is 2 otherwise 1 . Moreover, we also include the heavy Higgs decays with off-shell massive gauge bosons, which allows four-body decays of the heavy Higgs, as:

$$
\Gamma^{\hat{h}}\left(V_{1}^{*} V_{2}^{*}\right)=\frac{1}{\pi^{2}} \int_{0}^{m_{\hat{h}}^{2}} \frac{d s_{1} m_{V_{1}} \Gamma_{V_{1}}}{\left(s_{1}-m_{V_{1}}^{2}\right)^{2}+m_{V_{1}}^{2} \Gamma_{V_{1}}^{2}} \int_{0}^{\left(m_{\hat{h}}-\sqrt{s_{1}}\right)^{2}} \frac{d s_{2} m_{V_{2}} \Gamma_{V_{2}}}{\left(s_{2}-m_{V_{2}}^{2}\right)^{2}+m_{V_{2}}^{2} \Gamma_{V_{2}}^{2}} \Gamma^{\hat{h}}\left(V_{1} V_{2}\right)
$$


where $s_{1,2}$ are the squared invariant masses of the intermediate gauge bosons and $\Gamma_{V}$ being their total decay widths.

The partial decay widths of the twin Higgs to massless gauge bosons of the SM are,

$$
\begin{aligned}
\Gamma^{\hat{h}}(g g) & =\frac{G_{\mathrm{F}} \alpha_{\mathrm{QCD}}^{2} m_{\hat{h}}^{3}}{64 \sqrt{2} \pi^{3}}\left|\sum_{i=t, b} F_{1 / 2}\left(\tau_{i}\right)\right|^{2} g_{\hat{h}}^{2}, \\
\Gamma^{\hat{h}}(\gamma \gamma) & =\frac{G_{\mathrm{F}} \alpha_{\mathrm{EM}}^{2} m_{\hat{h}}^{3}}{128 \sqrt{2} \pi^{3}}\left|\sum_{i=t, b} e_{i}^{2} N_{c}^{i} F_{1 / 2}\left(\tau_{i}\right)+F_{1}\left(\tau_{W}\right)\right|^{2} g_{\hat{h}}^{2}, \\
\Gamma^{\hat{h}}(Z \gamma) & =\frac{G_{\mathrm{F}} \alpha_{\mathrm{EM}} m_{\hat{h}} m_{Z}^{2}}{64 \pi^{4}} \sin ^{2} \theta_{\mathrm{W}} \cos ^{2} \theta_{\mathrm{W}}\left(1-\frac{m_{Z}^{2}}{m_{\hat{h}}^{2}}\right)^{3}\left|\sum_{i=t, b} A_{1 / 2}+A_{1}\right|^{2} g_{\hat{h}}^{2},
\end{aligned}
$$

where the triangle loop functions $F_{1 / 2}, F_{1}, A_{F}$ and $A_{W}$ are adopted from the Higgs Hunter's Guide [74] and for completeness we collect them below.

It is straightforward to obtain the heavy twin Higgs partial decay widths to the twin gauge bosons from the above relations by interchanging $V \rightarrow \hat{V}, g_{\hat{h}} \rightarrow \hat{g}_{\hat{h}}, G_{\mathrm{F}} \rightarrow \hat{G}_{\mathrm{F}}, \alpha_{\mathrm{EM}} \rightarrow$ $\hat{\alpha}_{\mathrm{EM}}, \alpha_{\mathrm{QCD}} \rightarrow \hat{\alpha}_{\mathrm{QCD}} F_{1 / 2} \rightarrow \hat{F}_{1 / 2}, F_{1} \rightarrow \hat{F}_{1}, A_{F} \rightarrow \hat{A}_{F}$ and $A_{W} \rightarrow \hat{A}_{W}$.

Heavy Higgs decays into SM di-Higgs. The heavy twin Higgs partial decay width to a pair of the SM Higgs is,

$$
\Gamma^{\hat{h}}(h h)=\frac{g_{\hat{h} h h}^{2}}{32 \pi} \frac{1}{m_{\hat{h}}} \sqrt{1-\frac{4 m_{h}^{2}}{m_{\hat{h}}^{2}}},
$$

where the trilinear coupling $g_{\hat{h} h h}$ is given in (2.19).

The loop functions. Following the notations of the Higgs Hunter's Guide [74], the form factors $F_{1 / 2}, F_{1}, A_{F}$ and $A_{W}$ are

$$
\begin{aligned}
& F_{1 / 2}\left(\tau_{i}\right)=-2 \tau_{i}\left[1+\left(1-\tau_{i}\right) f\left(\tau_{i}\right)\right], \quad F_{1}\left(\tau_{i}\right)=2+3 \tau_{i}+3 \tau\left(2-\tau_{i}\right) f\left(\tau_{i}\right), \\
& A_{1 / 2}\left(\tau_{i}, \kappa_{i}\right)=\frac{-e_{i} N_{c}^{i}}{\sin \theta_{\mathrm{W}} \cos \theta_{\mathrm{W}}}\left(1-4 e_{i} \sin ^{2} \theta_{\mathrm{W}}\right)\left[\mathcal{I}_{1}\left(\tau_{i}, \kappa_{i}\right)-\mathcal{I}_{2}\left(\tau_{i}, \kappa_{i}\right)\right], \\
& A_{1}\left(\tau_{i}, \kappa_{i}\right)=-\frac{\cos \theta_{\mathrm{W}}}{\sin \theta_{\mathrm{W}}}\left[4\left(3-\frac{\sin ^{2} \theta_{\mathrm{W}}}{\cos ^{2} \theta_{\mathrm{W}}}\right) \mathcal{I}_{2}\left(\tau_{W}, \kappa_{W}\right)\right. \\
&\left.\quad+\left(\left(1+2 / \tau_{W}\right) \frac{\sin ^{2} \theta_{\mathrm{W}}}{\cos ^{2} \theta_{\mathrm{W}}}-\left(5+2 / \tau_{W}\right)\right) \mathcal{I}_{1}\left(\tau_{W}, \kappa_{W}\right)\right],
\end{aligned}
$$

where $\tau_{i} \equiv 4 m_{i}^{2} / m_{\hat{h}}^{2}, \kappa_{i} \equiv 4 m_{i}^{2} / m_{Z}^{2}$, and

$$
\begin{aligned}
& \mathcal{I}_{1}\left(\tau_{i}, \kappa_{i}\right)=\frac{\tau_{i} \kappa_{i}}{2\left(\tau_{i}-\kappa_{i}\right)}+\frac{\tau_{i}^{2} \kappa_{i}^{2}}{2\left(\tau_{i}-\kappa_{i}\right)^{2}}\left(f\left(\tau_{i}\right)-f\left(\kappa_{i}\right)\right)+\frac{\tau_{i}^{2} \kappa_{i}}{\left(\tau_{i}-\kappa_{i}\right)^{2}}\left(g\left(\tau_{i}\right)-g\left(\kappa_{i}\right)\right), \\
& \mathcal{I}_{2}\left(\tau_{i}, \kappa_{i}\right)=-\frac{\tau_{i} \kappa_{i}}{2\left(\tau_{i}-\kappa_{i}\right)}\left(f\left(\tau_{i}\right)-f\left(\kappa_{i}\right)\right),
\end{aligned}
$$




$$
\begin{aligned}
& f\left(\tau_{i}\right)=\left\{\begin{array}{llc}
\arcsin ^{2}\left(1 / \sqrt{\tau_{i}}\right), & \text { if } & \tau_{i} \geq 1, \\
-\frac{1}{4}\left[\ln \left(\frac{1+\sqrt{1-\tau_{i}}}{1-\sqrt{1-\tau_{i}}}\right)-i \pi\right]^{2}, & \text { if } & \tau_{i}<1,
\end{array}\right. \\
& g\left(\tau_{i}\right)=\left\{\begin{array}{lll}
\sqrt{\tau_{i}-1} \arcsin { }^{2}\left(1 / \sqrt{\tau_{i}}\right), & \text { if } & \tau_{i} \geq 1, \\
-\frac{\sqrt{1-\tau_{i}}}{2}\left[\ln \left(\frac{1+\sqrt{1-\tau_{i}}}{1-\sqrt{1-\tau_{i}}}\right)-i \pi\right]^{2}, & \text { if } & \tau_{i}<1 .
\end{array}\right.
\end{aligned}
$$

Note that the corresponding twin sector loop functions are straightforward to obtain from above generic formulae by appropriately replacing the masses, couplings, etc.

Open Access. This article is distributed under the terms of the Creative Commons Attribution License (CC-BY 4.0), which permits any use, distribution and reproduction in any medium, provided the original author(s) and source are credited.

\section{References}

[1] Z. Chacko, H.-S. Goh and R. Harnik, The Twin Higgs: Natural electroweak breaking from mirror symmetry, Phys. Rev. Lett. 96 (2006) 231802 [hep-ph/0506256] [INSPIRE].

[2] R. Barbieri, T. Gregoire and L.J. Hall, Mirror world at the large hadron collider, hep-ph/0509242 [INSPIRE].

[3] Z. Chacko, Y. Nomura, M. Papucci and G. Perez, Natural little hierarchy from a partially goldstone twin Higgs, JHEP 01 (2006) 126 [hep-ph/0510273] [INSPIRE].

[4] Z. Chacko, H.-S. Goh and R. Harnik, A Twin Higgs model from left-right symmetry, JHEP 01 (2006) 108 [hep-ph/0512088] [INSPIRE].

[5] N. Craig, A. Katz, M. Strassler and R. Sundrum, Naturalness in the Dark at the LHC, JHEP 07 (2015) 105 [arXiv: 1501.05310] [INSPIRE].

[6] M.J. Strassler and K.M. Zurek, Echoes of a hidden valley at hadron colliders, Phys. Lett. B 651 (2007) 374 [hep-ph/0604261] [INSPIRE].

[7] M.J. Strassler and K.M. Zurek, Discovering the Higgs through highly-displaced vertices, Phys. Lett. B 661 (2008) 263 [hep-ph/0605193] [INSPIRE].

[8] T. Han, Z. Si, K.M. Zurek and M.J. Strassler, Phenomenology of hidden valleys at hadron colliders, JHEP 07 (2008) 008 [arXiv:0712.2041] [INSPIRE].

[9] J. Kang and M.A. Luty, Macroscopic Strings and 'Quirks' at Colliders, JHEP 11 (2009) 065 [arXiv:0805.4642] [INSPIRE].

[10] J.E. Juknevich, D. Melnikov and M.J. Strassler, A Pure-Glue Hidden Valley I. States and Decays, JHEP 07 (2009) 055 [arXiv: 0903. 0883] [inSPIRE].

[11] J.E. Juknevich, Pure-glue hidden valleys through the Higgs portal, JHEP 08 (2010) 121 [arXiv: 0911.5616] [INSPIRE].

[12] G. Burdman, Z. Chacko, H.-S. Goh and R. Harnik, Folded supersymmetry and the LEP paradox, JHEP 02 (2007) 009 [hep-ph/0609152] [INSPIRE].

[13] A. Falkowski, S. Pokorski and M. Schmaltz, Twin SUSY, Phys. Rev. D 74 (2006) 035003 [hep-ph/0604066] [INSPIRE].

[14] S. Chang, L.J. Hall and N. Weiner, A Supersymmetric twin Higgs, Phys. Rev. D 75 (2007) 035009 [hep-ph/0604076] [INSPIRE]. 
[15] N. Craig and K. Howe, Doubling down on naturalness with a supersymmetric twin Higgs, JHEP 03 (2014) 140 [arXiv: 1312.1341] [INSPIRE].

[16] A. Katz, A. Mariotti, S. Pokorski, D. Redigolo and R. Ziegler, SUSY Meets Her Twin, JHEP 01 (2017) 142 [arXiv:1611.08615] [INSPIRE].

[17] M. Badziak and K. Harigaya, Supersymmetric D-term Twin Higgs, JHEP 06 (2017) 065 [arXiv: 1703.02122] [INSPIRE].

[18] M. Badziak and K. Harigaya, Minimal Non-Abelian Supersymmetric Twin Higgs, JHEP 10 (2017) 109 [arXiv:1707.09071] [InSPIRE].

[19] P. Batra and Z. Chacko, A Composite Twin Higgs Model, Phys. Rev. D 79 (2009) 095012 [arXiv: 0811.0394] [INSPIRE].

[20] N. Craig, S. Knapen and P. Longhi, Neutral Naturalness from Orbifold Higgs Models, Phys. Rev. Lett. 114 (2015) 061803 [arXiv: 1410.6808] [INSPIRE].

[21] M. Geller and O. Telem, Holographic Twin Higgs Model, Phys. Rev. Lett. 114 (2015) 191801 [arXiv: 1411.2974] [INSPIRE].

[22] R. Barbieri, D. Greco, R. Rattazzi and A. Wulzer, The Composite Twin Higgs scenario, JHEP 08 (2015) 161 [arXiv: 1501.07803] [INSPIRE].

[23] M. Low, A. Tesi and L.-T. Wang, Twin Higgs mechanism and a composite Higgs boson, Phys. Rev. D 91 (2015) 095012 [arXiv:1501. 07890] [INSPIRE].

[24] C. Csáki, M. Geller, O. Telem and A. Weiler, The Flavor of the Composite Twin Higgs, JHEP 09 (2016) 146 [arXiv: 1512.03427] [INSPIRE].

[25] N. Craig, S. Knapen and P. Longhi, The Orbifold Higgs, JHEP 03 (2015) 106 [arXiv: 1411.7393] [INSPIRE].

[26] G. Burdman, Z. Chacko, R. Harnik, L. de Lima and C.B. Verhaaren, Colorless Top Partners, a $125 \mathrm{GeV}$ Higgs and the Limits on Naturalness, Phys. Rev. D 91 (2015) 055007 [arXiv: 1411.3310] [INSPIRE].

[27] H. Beauchesne, K. Earl and T. Grégoire, The spontaneous $\mathbb{Z}_{2}$ breaking Twin Higgs, JHEP 01 (2016) 130 [arXiv: 1510.06069] [INSPIRE].

[28] J.-H. Yu, Radiative- $\mathbb{Z}_{2}$-breaking twin Higgs model, Phys. Rev. D 94 (2016) 111704 [arXiv: 1608.01314] [INSPIRE].

[29] N. Craig, S. Knapen, P. Longhi and M. Strassler, The Vector-like Twin Higgs, JHEP 07 (2016) 002 [arXiv: 1601.07181] [inSPIRE].

[30] R. Harnik, K. Howe and J. Kearney, Tadpole-Induced Electroweak Symmetry Breaking and PNGB Higgs Models, JHEP 03 (2017) 111 [arXiv:1603.03772] [INSPIRE].

[31] R. Barbieri, L.J. Hall and K. Harigaya, Minimal Mirror Twin Higgs, JHEP 11 (2016) 172 [arXiv: 1609.05589] [INSPIRE].

[32] Z. Chacko, N. Craig, P.J. Fox and R. Harnik, Cosmology in Mirror Twin Higgs and Neutrino Masses, JHEP 07 (2017) 023 [arXiv: 1611.07975] [INSPIRE].

[33] R. Barbieri, L.J. Hall and K. Harigaya, Effective Theory of Flavor for Minimal Mirror Twin Higgs, JHEP 10 (2017) 015 [arXiv: 1706. 05548] [INSPIRE].

[34] J. Serra and R. Torre, The Brother Higgs, arXiv:1709.05399 [InSPIRE].

[35] C. Csáki, T. Ma and J. Shu, Trigonometric Parity for the Composite Higgs, arXiv: 1709.08636 [INSPIRE]. 
[36] D. Buttazzo, F. Sala and A. Tesi, Singlet-like Higgs bosons at present and future colliders, JHEP 11 (2015) 158 [arXiv: 1505.05488] [INSPIRE].

[37] S. Fichet, G. von Gersdorff, E. Pontón and R. Rosenfeld, The Excitation of the Global Symmetry-Breaking Vacuum in Composite Higgs Models, JHEP 09 (2016) 158 [arXiv: 1607.03125] [INSPIRE].

[38] S. Fichet, G. von Gersdorff, E. Pontón and R. Rosenfeld, The Global Higgs as a Signal for Compositeness at the LHC, JHEP 01 (2017) 012 [arXiv: 1608.01995] [INSPIRE].

[39] D. Curtin and C.B. Verhaaren, Discovering Uncolored Naturalness in Exotic Higgs Decays, JHEP 12 (2015) 072 [arXiv: 1506.06141] [INSPIRE].

[40] C. Csáki, E. Kuflik, S. Lombardo and O. Slone, Searching for displaced Higgs boson decays, Phys. Rev. D 92 (2015) 073008 [arXiv: 1508.01522] [INSPIRE].

[41] Z. Chacko, D. Curtin and C.B. Verhaaren, A Quirky Probe of Neutral Naturalness, Phys. Rev. D 94 (2016) 011504 [arXiv:1512.05782] [INSPIRE].

[42] A. Pierce, B. Shakya, Y. Tsai and Y. Zhao, Searching for Confining Hidden Valleys at the $L H C(b)$, arXiv:1708.05389 [INSPIRE].

[43] R. Contino, D. Greco, R. Mahbubani, R. Rattazzi and R. Torre, Precision Tests and Fine Tuning in Twin Higgs Models, Phys. Rev. D 96 (2017) 095036 [arXiv:1702.00797] [INSPIRE].

[44] S. Dawson et al., Working Group Report: Higgs Boson, arXiv:1310.8361 [INSPIRE].

[45] J.R. Espinosa, C. Grojean, M. Muhlleitner and M. Trott, First Glimpses at Higgs' face, JHEP 12 (2012) 045 [arXiv: 1207.1717] [INSPIRE].

[46] GFitter Group collaboration, M. Baak et al., The global electroweak fit at NNLO and prospects for the LHC and ILC, Eur. Phys. J. C 74 (2014) 3046 [arXiv:1407.3792] [INSPIRE].

[47] Z. Chacko, C. Kilic, S. Najjari and C.B. Verhaaren, Phenomenology of the Twin Photon, to appear (2018).

[48] C. Csáki, E. Kuflik and S. Lombardo, Viable Twin Cosmology from Neutrino Mixing, Phys. Rev. D 96 (2017) 055013 [arXiv:1703.06884] [INSPIRE].

[49] A. Ahmed, B.M. Dillon, B. Grzadkowski, J.F. Gunion and Y. Jiang, Implications of the absence of high-mass radion signals, Phys. Rev. D 95 (2017) 095019 [arXiv:1512.05771] [INSPIRE].

[50] LHC Higgs Cross Section Working Group, D. de Florian et al., Handbook of LHC Higgs Cross Sections: 4. Deciphering the Nature of the Higgs Sector, arXiv:1610.07922 [InSPIRE].

[51] ATLAS and CMS collaborations, Measurements of the Higgs boson production and decay rates and constraints on its couplings from a combined ATLAS and CMS analysis of the LHC pp collision data at $\sqrt{s}=7$ and $8 \mathrm{TeV}$, JHEP 08 (2016) 045 [arXiv:1606. 02266] [INSPIRE].

[52] A. Djouadi, J. Kalinowski and M. Spira, HDECAY: A Program for Higgs boson decays in the standard model and its supersymmetric extension, Comput. Phys. Commun. 108 (1998) 56 [hep-ph/9704448] [INSPIRE].

[53] ATLAS collaboration, Search for $W W / W Z$ resonance production in $\ell \nu q q$ final states in $p p$ collisions at $\sqrt{s}=13 \mathrm{TeV}$ with the ATLAS detector, ATLAS-CONF-2017-051 (2017) [INSPIRE]. 
[54] ATLAS collaboration, Search for heavy $Z Z$ resonances in the $\ell^{+} \ell^{-} \ell^{+} \ell^{-}$and $\ell^{+} \ell^{-} \nu \bar{\nu}$ final states using proton-proton collisions at $\sqrt{s}=13 \mathrm{TeV}$ with the ATLAS detector, ATLAS-CONF-2017-058 (2017) [INSPIRE].

[55] ATLAS collaboration, Searches for heavy $Z Z$ and $Z W$ resonances in the $\ell \ell q q$ and $\nu \nu q q$ final states in pp collisions at $\sqrt{s}=13 \mathrm{TeV}$ with the ATLAS detector, arXiv:1708.09638 [INSPIRE].

[56] CMS collaboration, Searches for invisible decays of the Higgs boson in pp collisions at $\sqrt{s}=7,8$ and $13 \mathrm{TeV}$, JHEP 02 (2017) 135 [arXiv:1610.09218] [INSPIRE].

[57] D. Curtin et al., Exotic decays of the 125 GeV Higgs boson, Phys. Rev. D 90 (2014) 075004 [arXiv: 1312.4992] [INSPIRE].

[58] H.-C. Cheng, S. Jung, E. Salvioni and Y. Tsai, Exotic Quarks in Twin Higgs Models, JHEP 03 (2016) 074 [arXiv: 1512.02647] [INSPIRE].

[59] H.-C. Cheng, E. Salvioni and Y. Tsai, Exotic electroweak signals in the twin Higgs model, Phys. Rev. D 95 (2017) 115035 [arXiv: 1612.03176] [INSPIRE].

[60] O. Buchmueller et al., Simplified Models for Displaced Dark Matter Signatures, JHEP 09 (2017) 076 [arXiv: 1704.06515] [INSPIRE].

[61] R.K. Ellis, W.J. Stirling and B.R. Webber, QCD and collider physics, Camb. Monogr. Part. Phys. Nucl. Phys. Cosmol. 8 (1996) 1 [InSPIRE].

[62] P. Schwaller, D. Stolarski and A. Weiler, Emerging Jets, JHEP 05 (2015) 059 [arXiv: 1502.05409] [INSPIRE].

[63] T. Sjöstrand et al., An Introduction to PYTHIA 8.2, Comput. Phys. Commun. 191 (2015) 159 [arXiv: 1410.3012] [INSPIRE].

[64] B. Lucini, M. Teper and U. Wenger, Glueballs and k-strings in $\mathrm{SU}(N)$ gauge theories: Calculations with improved operators, JHEP 06 (2004) 012 [hep-lat/0404008] [INSPIRE].

[65] S. Knapen, S. Pagan Griso, M. Papucci and D.J. Robinson, Triggering Soft Bombs at the LHC, JHEP 08 (2017) 076 [arXiv: 1612.00850] [INSPIRE].

[66] T. Cohen, M. Lisanti, H.K. Lou and S. Mishra-Sharma, LHC Searches for Dark Sector Showers, JHEP 11 (2017) 196 [arXiv:1707.05326] [INSPIRE].

[67] ATLAS collaboration, Search for long-lived neutral particles decaying in the hadronic calorimeter of ATLAS at $\sqrt{s}=13 \mathrm{TeV}$ in $3.2 \mathrm{fb}^{-1}$ of data, ATLAS-CONF-2016-103 (2016) [INSPIRE].

[68] ATLAS collaboration, Search for massive, long-lived particles using multitrack displaced vertices or displaced lepton pairs in pp collisions at $\sqrt{s}=8 \mathrm{TeV}$ with the ATLAS detector, Phys. Rev. D 92 (2015) 072004 [arXiv: 1504.05162] [INSPIRE].

[69] ATLAS collaboration, Search for long-lived, weakly interacting particles that decay to displaced hadronic jets in proton-proton collisions at $\sqrt{s}=8 \mathrm{TeV}$ with the ATLAS detector, Phys. Rev. D 92 (2015) 012010 [arXiv: 1504.03634] [INSPIRE].

[70] CMS collaboration, Search for Long-Lived Neutral Particles Decaying to Quark-Antiquark Pairs in Proton-Proton Collisions at $\sqrt{s}=8$ TeV, Phys. Rev. D 91 (2015) 012007 [arXiv: 1411.6530] [INSPIRE].

[71] CMS collaboration, Search for long-lived particles that decay into final states containing two electrons or two muons in proton-proton collisions at $\sqrt{s}=8 \mathrm{TeV}$, Phys. Rev. D 91 (2015) 052012 [arXiv: 1411.6977] [INSPIRE]. 
[72] LHCb collaboration, Search for Higgs-like bosons decaying into long-lived exotic particles, Eur. Phys. J. C 76 (2016) 664 [arXiv: 1609.03124] [INSPIRE].

[73] Z. Chacko, C. Kilic, S. Najjari and C.B. Verhaaren, Testing the Scalar Sector of the Twin Higgs Model at Colliders, arXiv:1711.05300 [INSPIRE].

[74] J.F. Gunion, H.E. Haber, G.L. Kane and S. Dawson, The Higgs Hunter's Guide, Front. Phys. 80 (2000) 1 [INSPIRE]. 